Quarterly Reviews of Biophysics 10, 3 (1977), pp. 353-418.

Printed in Great Britain

\title{
Deuterium magnetic resonance: theory and application to lipid membranes
}

\author{
JOACHIM SEELIG
}

Department of Biophysical Chemistry, Biocenter of the University of Basel, Klingelbergstrasse 7o, $\mathrm{CH}-4{ }^{\circ}{ }^{6} \mathrm{Basel}$, Switzerland

I. INTRODUCTION

II. Theory of DeUterium Magnetic Resonance

A. Deuterium magnetic resonance in the absence of molecular motion

r. General theory $35^{8}$

2. Rotation of the coordinate system 359

3. Principal coordinate system 360

4. Energy levels $3^{62}$

5. Lineshapes of polycrystalline samples $3^{6} 4$

B. Deuterium magnetic resonance of liquid crystals

I. Anisotropic motions in liquid crystals 369

2. Deuterium-order parameters in planar-oriented liquid crystals 373

3. Lineshapes for random and cylindrical distributions of liquid crystalline microdomains 377

4. Anisotropic rotation of $C D_{2}$ and $C D_{3}$ groups 383

5. Deuterium quadrupole relaxation in anisotropic media

III. APPlication OF DEUTERIUM MAgnetic Resonance TO LIPID MEMBRANES

A. Lipid bilayers composed of soap molecules 388

B. Phospholipid bilayers 393

I. Hydrocarbon region 394

2. Structure and flexibility of the polar head groups 405 


\section{INTRODUCTION}

Proton and carbon-I $3 \mathrm{nmr}$ spectra of unsonicated lipid bilayers and biological membranes are generally dominated by strong proton-proton and proton-carbon dipolar interactions. As a result the spectra contain a large number of overlapping resonances and are rather difficult to analyse. Nevertheless, important information on the structure and dynamic behaviour of lipid systems has been provided by these techniques (Wennerström \& Lindblom, 1977).

Deuterium magnetic resonance of membraneous systems differs from conventional proton or carbon- $13 \mathrm{nmr}$ by the following principal features. (I) By means of an appropriate chemical synthesis some hydrogen atoms in a membrane molecule are replaced by deuterium. (2) The deuterium $\mathrm{nmr}$ spectrum of a partially deuterated molecule consists of only a few resonances, which can be assigned unambiguously. (3) In deuterium $\mathrm{nmr}$ the dipolar couplings are much reduced compared to the corresponding proton spectra, owing to the smaller magnetic moment of the deuteron (magnetogyric ratios $\gamma_{\mathrm{D}} / \gamma_{\mathrm{H}}=\mathrm{I} / 6 \cdot 5$ ). (4) Deuterium nmr easily detects anisotropic motions. For a rapid isotropic motion the deuterium $\mathrm{nmr}$ spectrum of a deuteron consists of one single line, while for an anisotropic motion each deuteron contributes a doublet due to the quadrupole moment of the deuterium nucleus. The doublet spacing, $\Delta \nu_{Q}$, depends on the degree of anisotropy and on the orientation of the deuteron with respect to the molecular symmetry axis. In orientated samples the quadrupole splitting depends further on the angle between the magnetic field and the axis of motional averaging (director-axis).

Some of the properties of deuterium $\mathrm{nmr}$ are illustrated in Fig. I, where a small molecule, ethanol, is dissolved in a nematic liquid crystal. The ethanol participates in the anisotropic motion of the liquid crystalline solvent and the proton-proton dipolar interactions are only partially averaged out. The proton spectrum is complex and consists of many resonances, even though only a few protons contribute to it (Fig. I A). The analysis of this spectrum has been given by Emsley, Lindon \& Tabony $(1973 a, b)$. Compared to the proton spectrum the deuterium spectrum of $\mathrm{CH}_{3} \mathrm{CD}_{2} \mathrm{OH}$ in a nematic liquid crystal is much simpler (Fig. I B). The two deuterons in $\mathrm{CH}_{3} \mathrm{CD}_{2} \mathrm{OH}$ are equivalent and the $\mathrm{CD}_{2}$ group gives rise to just one doublet, the doublet spacing being directly related to the anisotropy of motion. The dominant feature of Fig. I B is thus the quadrupole interaction, while dipolar interactions can be 


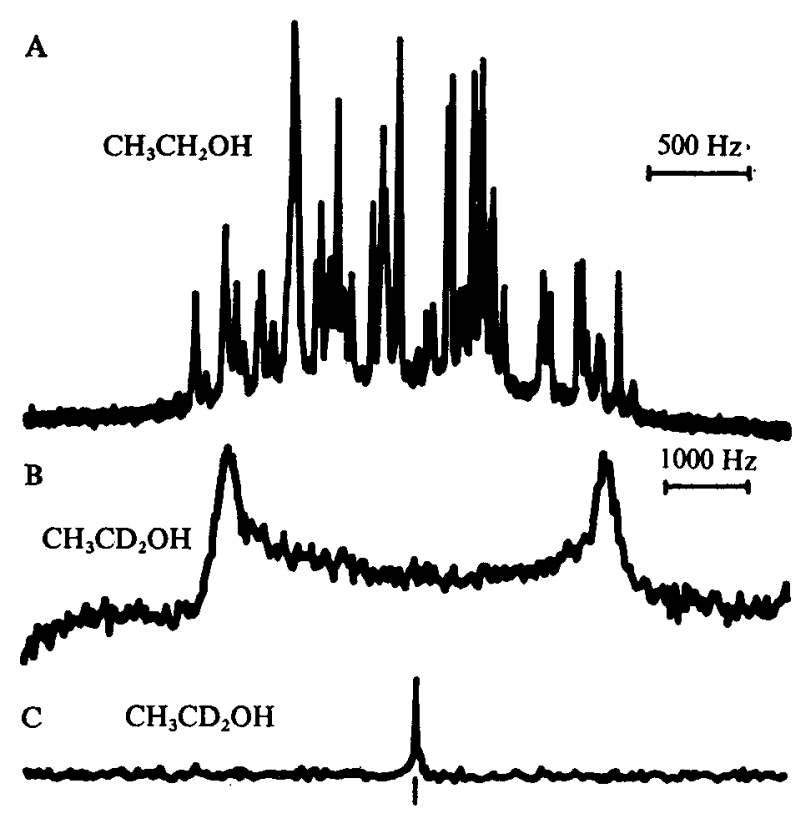

Fig. r. (A) Proton nmr spectrum of $\mathrm{CH}_{3} \mathrm{CH}_{2} \mathrm{OH}$ dissolved in a nematic liquid crystal (Emsley, Lindon \& Tabony, 1973a; reprinted with permission from the copyright owner). (B) Deuterium nmr spectrum of $\mathrm{CH}_{3} \mathrm{CD}_{2} \mathrm{OH}$ dissolved in a nematic liquid crystal. (C) Deuterium nmr spectrum of $\mathrm{CH}_{3} \mathrm{CD}_{2} \mathrm{OH}$ dissolved in $\mathrm{CHCl}_{3}$.

neglected. If the $\mathrm{CH}_{3} \mathrm{CD}_{2} \mathrm{OH}$ is dissolved in $\mathrm{CHCl}_{3}$ the motion is isotropic and the quadrupole splitting collapses (Fig. $\mathrm{IC}$ ). For isotropic solutions the deuterium $\mathrm{nmr}$ spectra are similar to conventional proton $\mathrm{nmr}$. In particular, the deuterium chemical shifts are almost the same as the proton chemical shifts. On the other hand, the ratio of isotropic coupling constant to chemical shift is still reduced by a factor of 6.5 and the analysis of the deuterium nmr spectra is straightforward. The characteristics and advantages of deuterium $\mathrm{nmr}$ in solution have recently been reviewed by Diehl (1974) and by Mantsch, Saitô \& Smith (1977).

In considering a pure lipid membrane one is primarily interested in the structure of the membrane, that is the average orientation of the hydrocarbon chains and the polar groups and the amplitudes of fluctuation of the lipid segments. The second point of interest concerns the dynamical properties of the bilayer, that is the rate of segmental motions and the rate of diffusion of the lipids within each half layer. In terms of experimental parameters characteristic of magnetic resonance techniques 
TABLE I. Deuteron quadrupole coupling constants

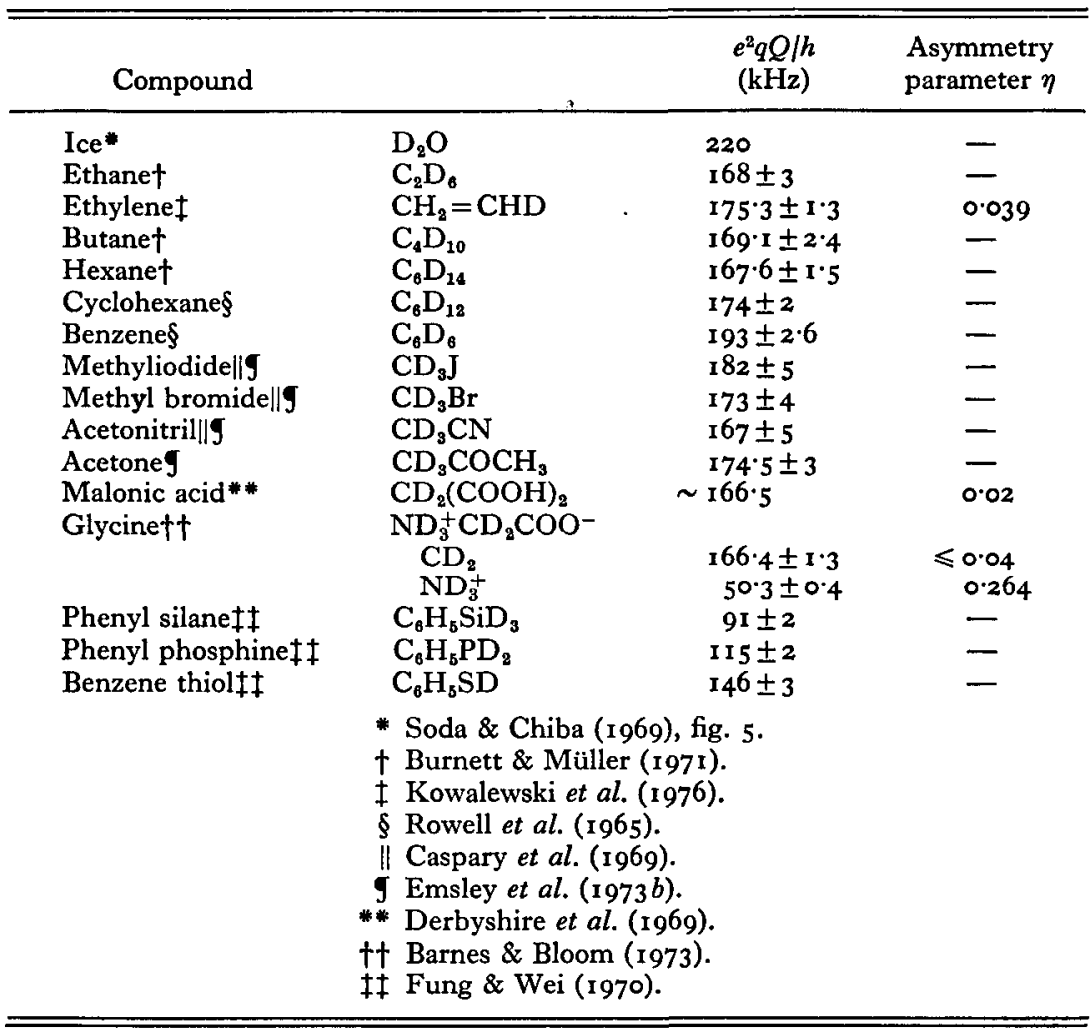

these problems are solved if the segmental order parameters and the $T_{1}$ and $T_{2}$ relaxation times are measured and if a consistent molecular interpretation of the experimental data can be provided. In both respects deuterium nmr of selectively deuterated lipids offers distinct advantages. The order parameter $S_{\mathrm{CD}}$ of a deuterium bond vector can readily be evaluated from the quadrupole splitting $\Delta \nu_{Q}$. For a sample of randomly distributed bilayers ('powder'-type spectra) the order parameter $S_{\mathrm{CD}}$ is related to the doublet spacing according to (cf. §II)

$$
\Delta v_{Q}=(3 / 4)\left(e^{2} q Q / h\right) S_{\mathrm{CD}},
$$

where $\left(e^{2} q Q / h\right)$ is the static quadrupole splitting constant. For paraffinic $\mathrm{C}-\mathrm{D}$ bonds $\left(e^{2} q Q / h\right)$ has been determined to be $170 \mathrm{kHz}$ (Burnett \& Müller, I97I). Deuterium quadrupole coupling constants for some other types of deuterium bonds are summarized in Table I (see also Barnes, 
I974). The order parameter can then be interpreted in terms of statistical models for the bilayer structure. In addition, the interpretation of deuterium $T_{1}$ and $T_{2}$ relaxation times in deuterium $\mathrm{nmr}$ is less complicated than in proton or carbon- $\mathrm{I} 3 \mathrm{nmr}$ since the dominant relaxation mechanism is the quadrupole interaction. Relaxation time measurements will not be discussed in greater detail in this article however.

Notwithstanding essential experimental differences deuterium magnetic resonance yields similar information to that given by spin label epr. Using spin labels the order parameter is easily determined from the anisotropy of the hyperfine splitting, while the correlation times can be deduced from the linewidth (cf. Seelig, 1976). Compared to spin label epr where the attachment of the bulky nitroxide spin label group is a prerequisite for the measurements, deuterium labelling has the important advantage that deuterium substitution does not alter the molecular structure. Deuterium labelling is thus a non-perturbing probe technique. Important qualitative and quantitative differences between deuterium $\mathrm{nmr}$ and spin label epr have been observed for a number of bilayer systems, which clearly must be attributed to the perturbation of the bilayer structure by the spin label group.

The drawbacks of deuterium magnetic resonance should also be recognized. In order to take full advantage of deuterium nmr, selectively deuterated lipids or other deuterated membrane constituents must be available. The synthesis of such molecules may pose a serious problem for some investigations. The second disadvantage is the rather low sensitivity associated with deuterium $\mathrm{nmr}$. At present, specimens with deuterium contents of at least $0 \cdot \mathrm{I}-\mathrm{I} \mathrm{mg}$ are required for successful measurements. For practical purposes this means that the percentage of selectively deuterated molecules must be rather high.

The application of deuterium nmr to liquid crystals and membranes is a comparatively recent method. The literature contains a number of reports on the hydration of phospholipid bilayers and other macromolecular systems with $\mathrm{D}_{2} \mathrm{O}$ (e.g. Finer, 1973, and references quoted therein). The use of selectively deuterated molecules combined with deuterium $\mathrm{nmr}$ has been introduced in the investigation of thermotropic liquid crystals (nematic phase) by Rowell et al. (1965). Lyotropic liquid crystals have first been studied using bilayers composed of perdeuterated lipids (Oldfield, Chapman \& Derbyshire, 1971; Charvolin, Manneville \& Deloche, 1973). Only recently has the development of pulse Fouriertransform techniques made it possible to detect deuterium resonance 
signals even at low concentrations of deuterium, thus allowing measurements with selectively deuterated lipids (Saitô, Schreier-Muccillo \& Smith, 1973; Seelig \& Niederberger, I974 $a, b$; Seelig \& Seelig, 1974 $a, b$; Niederberger \& Seelig, 1974; Fujiwara et al. 1974; Stockton et al. 1974; Gally, Niederberger \& Seelig, 1975; Seelig \& Seelig, r975; Reeves \& Tracey, 1975; Stockton et al. 1976; Gally, Seelig \& Seelig, 1976; Seelig \& Gally, 1976; Seelig \& Seelig, 1977; Seelig, Gally \& Wohlgemuth, 1977). Since the preparation of selectively deuterated compounds often requires elaborate synthesis, the use of commercially available perdeuterated fatty acids as lipid probes remains an attractive alternative, even though the assignment of the resonances is not unambiguous (Mely, Charvolin \& Keller, I975; Stockton \& Smith, 1976; Long \& Goldstein, 1976; Mely \& Charvolin, 1977; Davis \& Jeffrey, 1977). Before considering these experimental results a more detailed presentation of the theory of deuterium magnetic resonance will be given.

\section{Theory of DeUterium Magnetic Resonance}

Elementary introductions into the theory of electric quadrupole effects are found in the books of Slichter (1963) and Townes \& Schawlow (1955). A more extensive account of the subject has been given by Cohen $\&$ Reif (1957). Let us briefly summarize the essential aspects of the theory as far as they are pertinent to deuterium magnetic resonance.

\section{A. Deuterium magnetic resonance in the absence of molecular motion}

\section{General theory}

The energy of a deuterium nucleus in a magnetic field is composed of the magnetic energy $E_{M}$ and the quadrupole energy $E_{Q}$. The total Hamiltonian $\mathscr{H}$ may be written as

$$
\mathscr{H}=\mathscr{H}_{M}+\mathscr{H}_{Q} .
$$

$\mathscr{H}_{M}$ is the magnetic Hamiltonian which describes the interaction of the nuclear magnetic moment $\mu_{N}$ with the magnetic field $\mathbf{H}_{0}$.

$$
\mathscr{H}_{M}=-\mu_{N} \cdot \mathbf{H}_{0}=-g \beta_{N} \mathbf{I} \cdot \mathbf{H}_{0} .
$$

$I$ is the nuclear spin operator, $\beta_{N}$ the nuclear magneton, and $g$ the socalled $g$ factor. The quadrupole Hamiltonian $\mathscr{H}_{Q}$ arises from an electrostatic interaction of the nuclear quadrupole moment $\left(Q_{i k}\right)$ with the electric field gradient $\nabla E=\left(V_{i k}\right)$ at the position of the nucleus. The 
notations $\left(Q_{i k}\right)$ and $\left(V_{i k}\right)$ indicate that quadrupole moment and electric field gradient are second rank tensors. The quadrupole Hamiltonian is the scalar product of the two tensors. Different formulations of this product are possible, the most convenient being (cf. Slichter, 1963, p. I7I):

$$
\mathscr{H}_{Q}=\frac{e Q}{4 I(2 I-\mathrm{I})}\left[V_{0}\left(3 I_{z}^{2}-I^{2}\right)+V_{ \pm 1}\left(I_{\mp} I_{z}+I_{z} I_{\mp}\right)+V_{ \pm 2} I_{\mp}^{2}\right] .
$$

$Q$ is the (scalar) quadrupole moment $\left(Q_{\text {deuteron }}=2.875 \times 10^{-27} \mathrm{~cm}^{2}\right.$; Reid \& Vaida, I972), $e$ the elementary charge, and $I$ the nuclear spin ( $I=\mathrm{I}$ for deuterium). The following abbreviations are used in equation (4):

$$
\begin{aligned}
& V_{0}=V_{z z}, \\
& V_{ \pm 1}=V_{x z} \pm i V_{y z}, \\
& V_{ \pm 2}=\frac{1}{2}\left(V_{x x}-V_{y y} \pm 2 i V_{x y}\right) .
\end{aligned}
$$

The subscripts indicate second derivatives of the electrostatic potential $V$ with respect to the $x, y, z$ coordinate axes. The $I_{ \pm}$are the raising and lowering operators:

$$
I_{ \pm}=I_{x} \pm i I_{y}
$$

Equation (4) is the general formulation of the quadrupole Hamiltonian. It is valid for an arbitrary choice of the direction of the cartesian axes, provided that the $V_{i k}$ and the spin operators $I_{z}$ and $I_{ \pm}$are referred to the same coordinate system.

\section{Rotation of the coordinate system}

The electric field gradient tensor $\nabla \mathbf{E}=\left(V_{i k}\right)$ is usually known in a molecule fixed coordinate system. The spin operators $I_{x}, I_{y}$, etc., are, however, quantized along the laboratory fixed magnetic field. In order to apply equation (4) it is then necessary to rotate $\left(V_{i k}\right)$ from the molecule fixed reference frame into the laboratory fixed coordinate system. This is accomplished by successive rotations of $\left(V_{i k}\right)$ through the Eulerian angles $\alpha, \beta, \gamma$ (for definitions of $\alpha, \beta$, and $\gamma$ see Rose (I957), p. 50).

Cartesian basis. If the electric field gradient tensor is expressed in a cartesian basis the transformation is carried out by means of the orthogonal transformation matrix $\mathbf{M}=\left(M_{i k}\right)$.

$$
\begin{aligned}
& \nabla E^{\prime}=\mathbf{M V E M}^{-1}, \\
& \left(V_{i k}^{\prime}\right)=\left(M_{i k}\right)\left(V_{i k}\right)\left(M_{i k}\right)^{-1} .
\end{aligned}
$$


The transformation matrix $\mathbf{M}$ has the components (Rose, I957, p. 65)

$\mathbf{M}=\left(\begin{array}{l}\cos \alpha \cos \beta \cos \gamma-\sin \alpha \sin \gamma \\ -\cos \alpha \cos \beta \sin \gamma-\sin \alpha \cos \gamma \\ \cos \alpha \sin \beta\end{array}\right.$

$$
\left.\begin{array}{cc}
\sin \alpha \cos \beta \cos \gamma+\cos \alpha \sin \gamma & -\sin \beta \cos \gamma \\
-\sin \alpha \cos \beta \sin \gamma+\cos \alpha \cos \gamma & \sin \beta \sin \gamma \\
\sin \alpha \sin \beta & \cos \beta
\end{array}\right) .
$$

Spherical basis. An alternative possibility is to express the electric field gradient tensor $\left(V_{i k}\right)$ in terms of its irreducible components $V^{(2, m)}(m=0$, $\pm \mathrm{I}, \pm 2)$. The tensor elements $V^{(2, m)}$ are given in terms of their cartesian components as follows:

$$
\left.\begin{array}{ll}
V^{(2,0)}=V_{z z} & =V_{0}, \\
V^{(2, \pm 1)}= \pm \sqrt{\frac{2}{3}}\left(V_{x z} \pm i V_{y z}\right) & =\mp \sqrt{\frac{2}{3}} V_{ \pm 1}, \\
V^{(2, \pm 2)}=\sqrt{\frac{1}{6}}\left(V_{x x}-V_{y y} \pm 2 i V_{x y}\right) & =\sqrt{\frac{2}{3}} V_{ \pm 2} .
\end{array}\right\}
$$

The coordinate transformation is then accomplished by using Wigner rotation matrices (cf. Rose, 1957):

$$
V^{(2, m)^{\prime}}=\sum_{p=0, \pm 1, \pm 2} D_{p m}^{(2)}(\alpha \beta \gamma) V^{(2, p)}
$$

The elements $D_{p m}^{(2)}$ of the $\mathbf{D}^{(2)}$-matrix are listed in Table 2.

\section{Principal coordinate system}

The electrostatic field gradient tensor is a symmetric and traceless tensor. It may therefore be transformed to a principal coordinate system in which it is diagonal and takes the form

Since

$$
\mathrm{V}_{p}=\left(\begin{array}{lll}
V_{x x} & 0 & 0 \\
0 & V_{y y} & 0 \\
0 & 0 & V_{z z}
\end{array}\right)
$$

$$
V_{x x}+V_{y y}+V_{z z}=0
$$

it is convenient to define two new parameters

$$
\begin{aligned}
& \text { field gradient: } \quad \text { eq }=V_{z z}, \\
& \text { asymmetry parameter }: \eta=\frac{V_{x x}-V_{y y}}{V_{z z}} .
\end{aligned}
$$

With the additional stipulation that the $z(y)$ - direction is always 


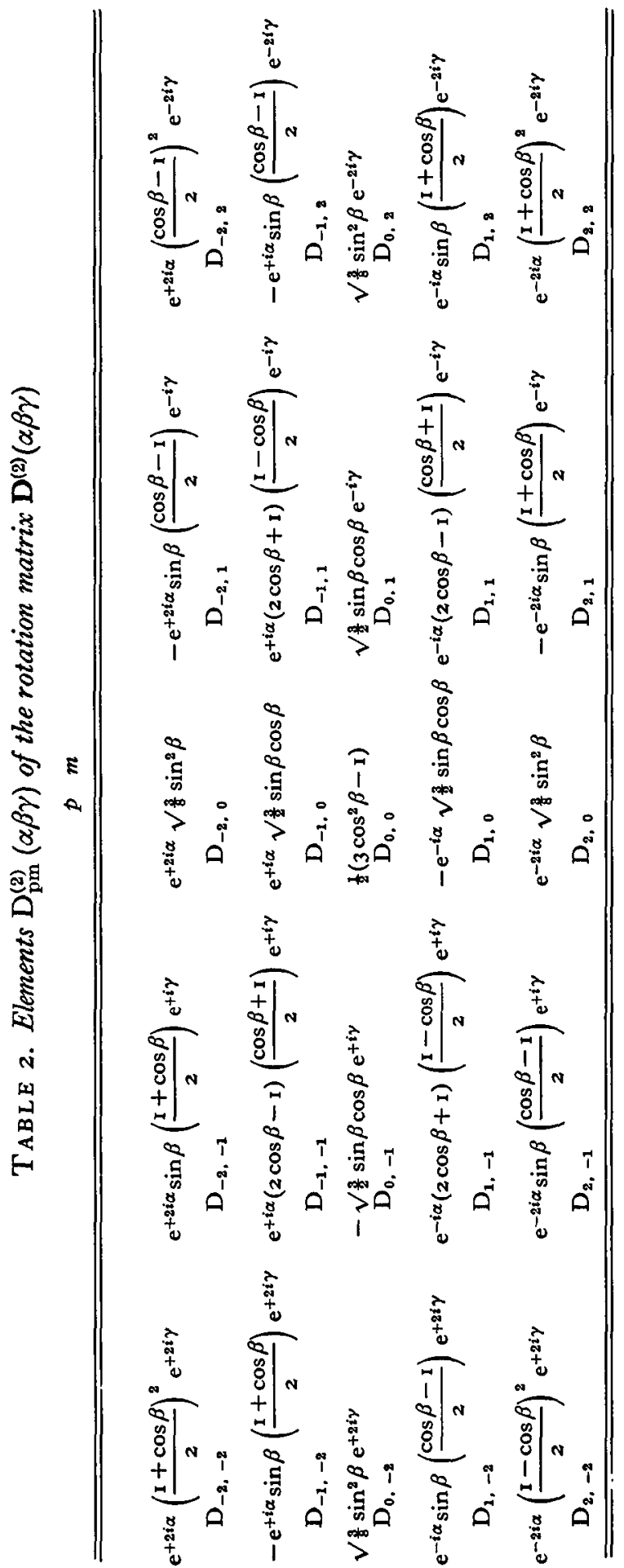


associated with the largest (smallest) field gradient $\left(V_{z z} \geqslant V_{x x} \geqslant V_{y y}\right)$ it immediately follows:

$$
0 \leqslant \eta \leqslant \mathrm{I} \text {. }
$$

Referred to the principal coordinate system the irreducible tensor elements $V_{P}^{(2, m)}$ are determined according to equation (9):

$$
\left.\begin{array}{ll}
V_{P}^{(2,0)}=V_{z z} & =e q, \\
V^{(2, \pm 1)}=0 & =0, \\
V^{(2, \pm 2)}=\sqrt{\frac{1}{6}}\left(V_{x x}-V_{y y}\right) & =\sqrt{\frac{1}{6} \eta e q .}
\end{array}\right\}
$$

4. Energy levels

In the case of the deuterium nucleus the quadrupolar energy $\left(E_{Q} \sim\right.$ $200 \mathrm{kHz}$ ) may be treated as a small perturbation of the magnetic energy ( $E_{M} \sim \mathrm{r}_{3} .8 \mathrm{MHz}$ at a field strength $H_{0}=2 \mathrm{I} \mathrm{kGs}$ ). In this 'high-field' approximation the energy levels of the total Hamiltonian are given to first order by

$$
E_{m}=-g \beta_{N} H_{0} m+\frac{e Q}{4 I(2 I-I)} V^{(2,0)}\left[3 m^{2}-I(I+\mathrm{I})\right]
$$

with $m=-I,-I+\mathrm{I}, \ldots, I-\mathrm{I}, I$. For deuterium with $I=\mathrm{I}$ the three energy levels may be written explicitly as:

$$
\left.\begin{array}{ll}
E_{+1}=-g \beta_{N} H_{0} & +\frac{1}{4} e Q V^{(2,0)}, \\
E_{0}= & -\frac{1}{2} e Q V^{(2,0)}, \\
E_{-1}=g \beta_{N} H_{0} & +\frac{1}{4} e Q V^{(2,0)} .
\end{array}\right\}
$$

The allowed transitions are given by the selection rule $\Delta m= \pm \mathrm{I}$ leading to the resonance energies

$$
\begin{aligned}
& h \nu_{+}=E_{-1}-E_{0}=g \beta_{N} H_{0}+\frac{3}{4} e Q V^{(2,0)}, \\
& h \nu_{-}=E_{0}-E_{+1}=g \beta_{N} H_{0}-\frac{3}{4} e Q V^{(2,0)} .
\end{aligned}
$$

Thus two resonance lines are observed in the deuterium nmr experiments, the frequency spacing of the two lines being the quadrupole splitting $\Delta \nu_{Q}$

$$
\Delta \nu_{Q}=\nu_{+}-\nu_{-}=\frac{3}{2} \frac{e Q}{h} \cdot V^{(2,0)} .
$$

This is the basic equation for the evaluation of dmr spectra. It is valid for a homogeneously orientated sample of deuterium spins, i.e. the pertinent deuterium bond vectors must be aligned parallel to each other. 
Under certain conditions the transition $\Delta m= \pm 2$ may become partially allowed. This double quantum transition has been discussed for deuterium $\mathrm{nmr}$ by Wennerström, Persson \& Lindman (1974) and more recently by Pines and co-workers (Pines et al. 1976; Vega \& Pines, 1977). It gives rise to a resonance line at the centre of the two $\Delta m= \pm$ I transitions, i.e. $h \nu(\Delta m= \pm 2)=$ $+g \beta_{N} H_{0}$. The double quantum transition has been proposed to explain the occurrence of a sharp central line in deuteron spectra of $\mathrm{D}_{2} \mathrm{O}$ in liquid crystalline phases.

A better physical feeling for equation (20) is obtained by discussing the spectral changes associated with the rotation of a single crystal containing only one type of deuterium spins in the magnetic field $H_{0}$. The simplest situation encountered is an alignment of the magnetic field $H_{0}$ parallel to one of the principal axes of the electric field gradient tensor. $V^{(2,0)}$ in equation (20) is then identical with the corresponding single crystal component $V_{x x}, V_{y y}$, or $V_{z z}$. By definition (p. 360) $V_{z z}=e q$ is the largest electric field gradient. The quadrupole splitting $\Delta \nu_{Q}$ therefore takes its maximum value for $H_{0}$ parallel to the $z$ principal axis:

$$
\Delta \nu_{Q}\left(H_{0} \| z\right)=\frac{3}{2} \frac{e Q}{h} V_{z z}=\frac{3}{2} \frac{e^{2} q Q}{h}
$$

$\left(e^{2} q Q / h\right)$ is generally referred to as the static quadrupolar coupling constant and some values for deuterium containing compounds are listed in Table I. Starting from $H_{0} \| z$ any arbitrary orientation of the single crystal is obtained by performing consecutively the following two rotations (Fig. 2). The single crystal is first rotated around the $z$-axis of the field gradient tensor by an angle $\phi$. This is then followed by a rotation around the new $y$-axis ( $\eta$-axis in fig. 2 ) by an angle $\theta$. The transformation is easily performed by applying the Wigner rotation matrix $D^{(2)}(\alpha \beta \gamma)$. The rotation angles $\alpha, \beta$, and $\gamma$ correspond to (cf. Rose, p. $5^{\circ}$ ):

$$
\alpha=\phi ; \quad \beta=\theta ; \quad \gamma=0 .
$$

The transformed element $V^{(2,0)}$ is then given according to equation (10):

$$
V^{(2,0)}=\sum_{p=0, \pm 1, \pm 2} D_{p, 0}^{(2)} V^{(2, p)},
$$

where the $V_{P}^{(2, p)}$ are given by equation (16) leading to

$$
\begin{aligned}
& V^{(2,0)}=\frac{1}{2}\left(3 \cos ^{2} \theta-\mathrm{I}\right) V_{z z}+\frac{1}{2} \sin ^{2} \theta \cos 2 \phi\left(V_{x x}-V_{y y}\right), \\
& V^{(2,0)}=\frac{1}{2} V_{z z}\left[\left(3 \cos ^{2} \theta-\mathrm{I}\right)+\eta \sin ^{2} \theta \cos 2 \phi\right] .
\end{aligned}
$$



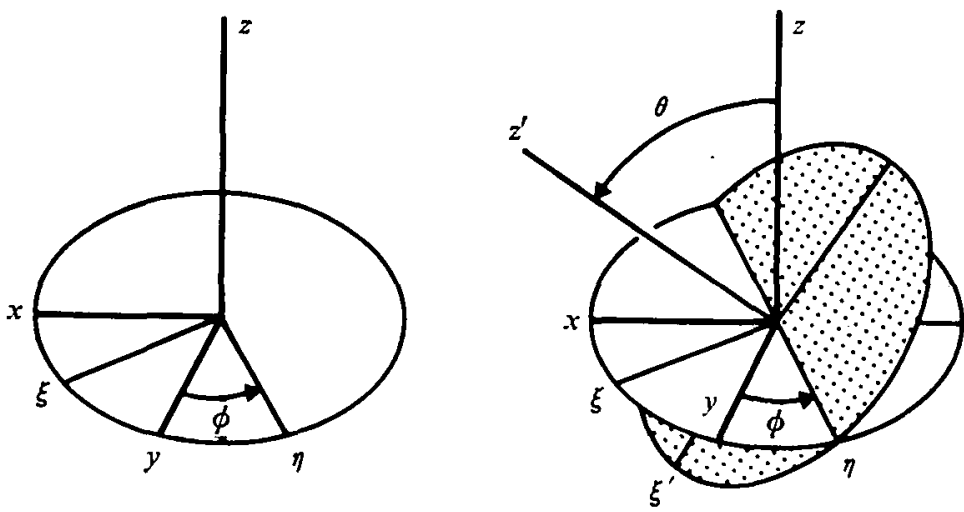

Fig. 2. Rotation of a single crystal in the magnetic field. $H_{0}$ is parallel to the $z$ axis. The first rotation of the crystal is performed around the $z$ axis, the second around the $\eta$ axis.

The variation of the deuterium quadrupolar splitting with $\theta$ and $\phi$ is thus found from combining equations (20) and (22):

$$
\Delta v_{Q}(\theta, \phi)=\frac{3}{2}\left(\frac{e^{2} q Q}{h}\right)\left(\frac{3 \cos ^{2} \theta-\mathrm{I}}{2}+\frac{1}{2} \eta \sin ^{2} \theta \cos 2 \phi\right) .
$$

For $V_{x x}=V_{y y}(\eta=0)$ this reduces to

$$
\Delta \nu_{Q}(\theta)=\frac{3}{2}\left(\frac{e^{2} q Q}{h}\right)\left(\frac{3 \cos ^{2} \theta-I}{2}\right) \text {. }
$$

Equation (24) shows that for an oriented sample with $\eta=0$ the quadrupole splitting collapses at $\cos ^{2} \theta=\frac{1}{3}$. This corresponds to an orientation angle of $\theta=5474^{\circ}$, which sometimes is referred to as the 'magic angle'.

\section{Line shapes of polycrystalline samples}

In the previous paragraph the energy levels and quadrupolar splittings have been derived for homogeneously oriented samples; all deuterium sites were assumed to have the same orientation with respect to the magnetic field. Single crystals are however difficult to prepare in many instances. We therefore consider a polycrystalline powder where the nuclear sites are randomly oriented with respect to the magnetic field $H_{0}$. The shape of this 'powder-type' spectrum is then the average over the resonances of all possible orientations of the nuclear spins.

Axial symmetry $(\eta=0)$. An analytical expression for the shape of the 'powder-type' spectrum is easy to derive when axial symmetry is present. 
Let us assume a uniform distribution of $N$ nuclei over a surface of a sphere of radius $r$. The number of nuclei per unit area is $N / 4 \pi r^{2}$. The fraction $\mathrm{d} N$ of nuclei oriented between $\theta$ and $\theta+\mathrm{d} \theta$ with respect to $H_{0}$ is given by the area of a zone of the sphere, $2 \pi r^{2} \sin \theta \mathrm{d} \theta$, multiplied by the spin surface density:

$$
\mathrm{d} N=\left(N / 4 \pi r^{2}\right) 2 \pi r^{2} \sin \theta \mathrm{d} \theta=(\mathrm{I} / 2) N \sin \theta \mathrm{d} \theta .
$$

The probability density $p(\theta)$ is thus given by

$$
\begin{gathered}
p(\theta)=(\mathrm{I} / 2) \sin \theta, \\
\int_{0}^{\pi} p(\theta) \mathrm{d} \theta=\mathrm{I} .
\end{gathered}
$$

According to equations ( 19 ) and (22) the resonance frequency shows the following dependence on the orientation angle $\theta$ :

$$
\nu_{ \pm}=\left(g \beta_{N} H_{0} / h\right) \pm(3 / 4)\left(e^{2} q Q / h\right)\left(\frac{3 \cos ^{2} \theta-I}{2}\right) .
$$

It is convenient to introduce a 'reduced' resonance frequency $\xi_{ \pm}$

$$
\xi_{ \pm}=\frac{\nu_{ \pm}-\left(g \beta_{N} H_{0} / h\right)}{(3 / 4)\left(e^{2} q Q / h\right)}= \pm \frac{3 \cos ^{2} \theta-\mathrm{I}}{2}
$$

where $\mathrm{I} \geqslant \xi_{+} \geqslant-\frac{1}{2}$ and $-\mathbf{I} \leqslant \xi_{-} \leqslant+\frac{1}{2}$. Since $\mathrm{nmr}$ spectra are recorded as a function of frequency, let us define the probability function $p(\xi)$ so that $p(\xi) \mathrm{d} \xi$ describes the fraction of spins with the reduced resonance frequency between $\xi$ and $\xi+\mathrm{d} \xi$. The two probability densities $p(\theta)$ and $p(\xi)$ are related to each other according to

$$
p(\xi)=p(\theta) \frac{\mathrm{d} \theta}{\mathrm{d} \xi}=\frac{1}{2} \sin \theta \frac{\mathrm{d} \theta}{\mathrm{d} \xi}=-\frac{\mathrm{I}}{2} \frac{\mathrm{d} \cos \theta}{\mathrm{d} \xi} .
$$

Now the deuterium spectrum contains two resonances $\xi_{+}$and $\xi_{-}$, so that

$$
p(\xi)=p\left(\xi_{+}\right)+p\left(\xi_{-}\right) \text {. }
$$

Combining equations (29) and (30) it then follows

$$
p\left(\xi_{ \pm}\right) \propto \frac{\mathrm{I}}{\sqrt{\left( \pm 2 \xi_{ \pm}+\mathrm{I}\right)}}
$$

The probability densities $p\left(\xi_{+}\right), p\left(\xi_{-}\right)$and $p(\xi)$ are plotted in Fig. 3 . $p(\xi)$ diverges at frequencies $\xi= \pm \frac{1}{2}$, which correspond to nuclear sites inclined at $\theta=90^{\circ}$ with respect to $H_{0}$. 


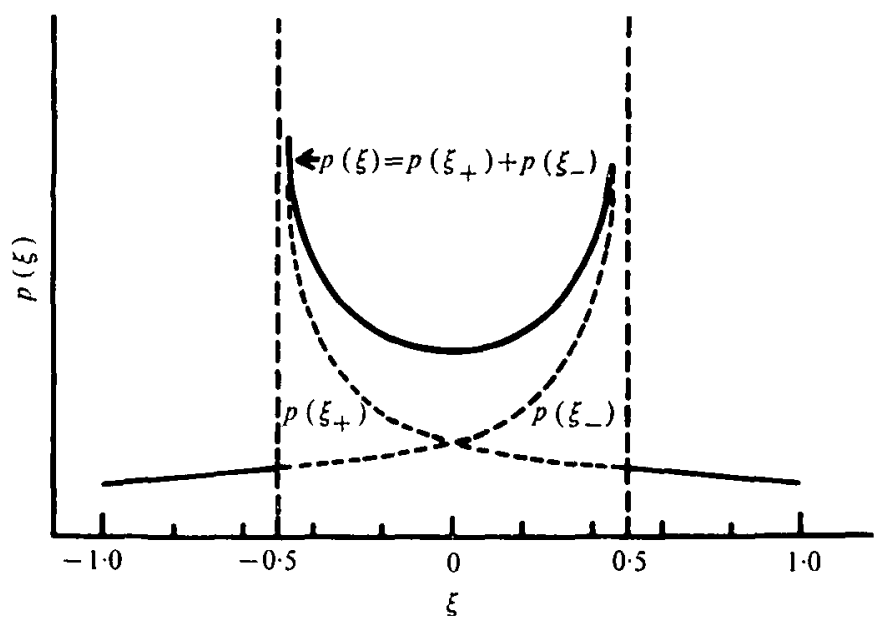

Fig. 3. Theoretical powder pattern for a deuterium nucleus in a symmetric electric field gradient $(\eta=0)$. The dashed lines show the individual components of the $m=-\mathrm{I} \leftrightarrow m=\circ\left(\xi_{+}\right)$and $m=\circ \leftrightarrow m=+_{\text {I }}\left(\xi_{-}\right)$transition, while the solid line indicates the sum of the two components.

To each resonance frequency $\xi_{ \pm}$there corresponds a transition probability $I\left(\xi_{t}\right)$. As a first-order approximation let us assume that $I\left(\xi_{ \pm}\right)$is equal for both deuterium transitions and independent of the nuclear orientation. The total absorption intensity $S(\xi)$ is then simply

$$
S(\xi)=I p(\xi)
$$

where $I$ is a constant. The absorption intensity is thus linearly related to $p(\xi)$ and the shape of the powder-type spectrum can be read off directly from Fig. 3. For practical purposes the most important feature of Fig. 3 is the frequency spacing of the two most intense peaks at $\xi=+\frac{1}{2}$ and $\xi=-\frac{1}{2}$, which is $\Delta \xi_{\text {powder }}=\mathrm{I}$ or

$$
\Delta \nu_{\text {powder }}=(3 / 4)\left(e^{2} q Q / h\right) \text {. }
$$

The static quadrupolar coupling constant $\left(e^{2} q Q / h\right)$ can therefore easily be determined from polycrystalline samples.

$\mathrm{Nmr}$ resonance lines are generally broadened due to magnetic interactions between neighbouring nuclei. Quite often the shape of an individual resonance line centred at frequency $\xi^{*}$ can be approximated by a normalized Gaussian of the form

$$
I\left(\xi-\xi^{*}\right)=\frac{\mathrm{I}}{\sqrt{(2 \pi) \sigma}} \exp \left[-\left(\xi-\xi^{*}\right)^{2} / 2 \sigma^{2}\right]
$$



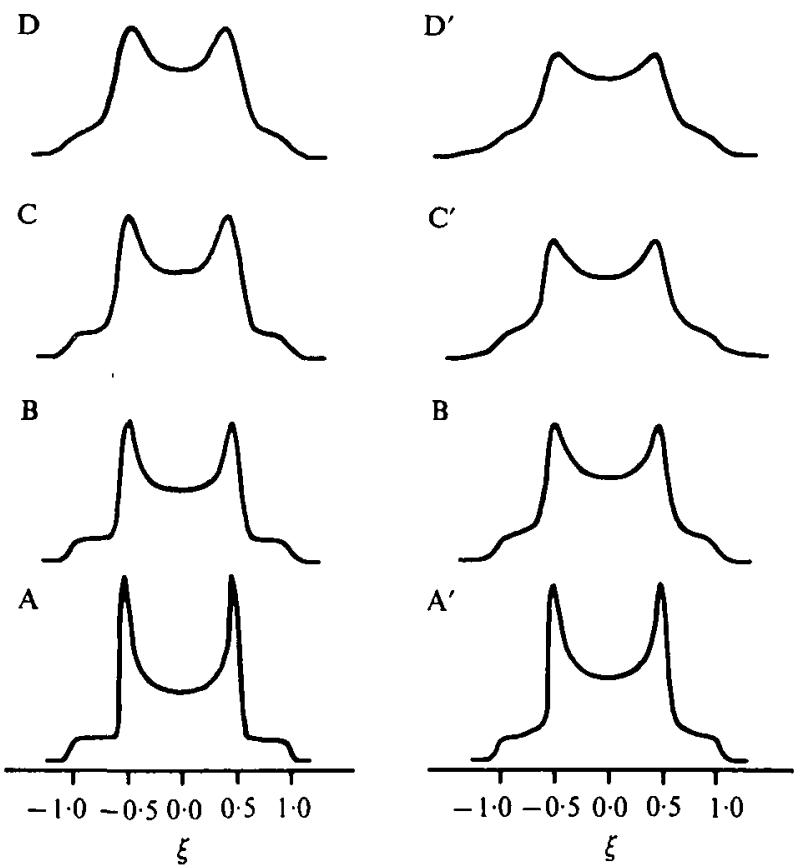

Fig. 4. The same spectrum as Fig. 3 but corrected for a finite linewidth $\sigma$. A Gaussian (Lorentzian) lineshape with a constant line-width $\sigma$ was assumed for all spectra $A-D\left(A^{\prime}-D^{\prime}\right)$. The figure illustrates the influence of $\sigma$ on the shape of the spectrum. A, $A^{\prime}, \sigma=0.025 ; B, B^{\prime}, \sigma=0.05 ; C, C^{\prime}, \sigma=0.075$; $\mathrm{D}, \mathrm{D}^{\prime}, \sigma=\mathrm{o} \cdot \mathrm{I}$.

Another possibility is to assume a Lorentzian line shape

$$
I\left(\xi-\xi^{*}\right)=\frac{\mathrm{I}}{\pi \sigma} \frac{\mathrm{I}}{\mathrm{I}+\left(\xi-\xi^{*}\right)^{2} / \sigma^{2}} .
$$

For a Gaussian line shape the linewidth at half-height is given by

$$
(\delta \xi)_{\frac{1}{2}}=\sigma \sqrt{ }\left(2^{3} \ln 2\right)=2 \cdot 35 \sigma .
$$

In a powder-type spectrum the total absorption $S(\xi)$ at a frequency $\xi$ is given by the convolution of the lineshape function $I\left(\xi-\xi^{*}\right)$ with the corresponding probability function $p\left(\xi^{*}\right)$

$$
S(\xi)=\int_{-\infty}^{+\infty} I\left(\xi-\xi^{*}\right) p\left(\xi^{*}\right) \mathrm{d} \xi^{*}
$$

Computer calculated 'powder-type' spectra $S(\xi)$ based on the assumption of Gaussian and Lorentzian line shapes are shown in Fig. 4. The spectra are computed for varying values of $\sigma$ and Fig. 4 demonstrates 

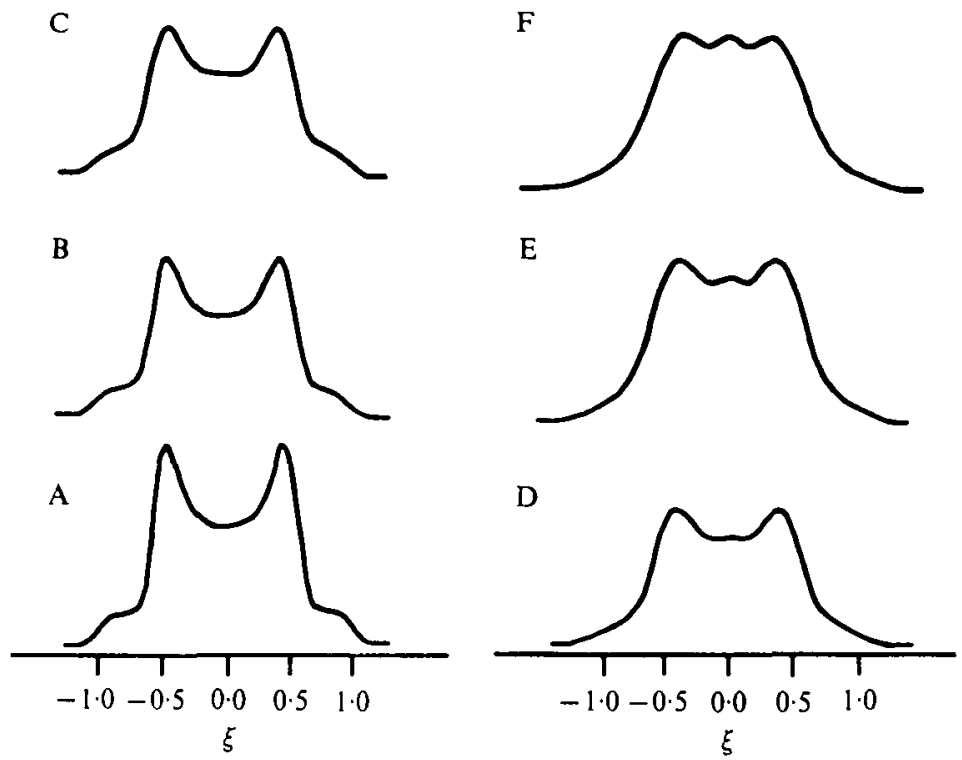

Fig. 5. The same spectrum as Fig. 3 but corrected for angular dependent dipolar effects. As in Fig. 4A-D, the lineshape was assumed to be a normalized Gaussian but this time the linewidth was approximated by

$$
\sigma=\sigma_{0}+\sigma_{1}\left|\left(3 \cos ^{2} \theta-1\right) / 2\right| \text {. }
$$

The figure illustrates the influence of $\sigma_{1}: \mathrm{A}, \sigma_{1}=0 ; \mathrm{B}, \sigma_{1}=0.025 ; \mathrm{C}$, $\sigma_{1}=0.05 ; \mathrm{D}, \sigma_{1}=0.1 ; \mathrm{E}, \sigma_{1}=0.15 ; \mathrm{F}, \sigma_{1}=0.2$. In all spectra $\sigma_{0}=0.075$.

that the peak-to-peak separation in the powder pattern approaches its theoretical value of $\Delta \xi=\mathbf{I}$ only for rather sharp lines, i.e. $(\delta \xi)_{\frac{1}{2}}<0 \cdot \mathrm{I}$. For broad resonances the peak-to-peak width of the powder pattern is found to be distinctly smaller than predicted by equation (34).

An additional complication occurs if the line shape $I\left(\xi-\xi^{*}\right)$ becomes dependent on the angle of orientation. Assuming essentially dipolar interactions the above formalism may be extended by introducing an angular dependent $\sigma$ parameter according to

$$
\sigma=\sigma_{0}+\sigma_{1}\left|\left(3 \cos ^{2} \theta-\mathrm{I}\right) / 2\right|,
$$

where $\sigma_{0}$ and $\sigma_{1}$ are empirical parameters. A series of computer calculated spectra based on Gaussian line shapes with various values for the parameter $\sigma_{1}$ is shown in Fig. 5. For large values of $\sigma_{1}$ the two absorption peaks at $\xi= \pm \frac{1}{2}$ are almost completely smoothed out and a new peak emerges at $\xi=0$ (Fig. $5 \mathrm{E}, \mathrm{F}$ ).

Asymmetric field gradient tensor $\left(V_{i k}\right)(\eta \neq 0)$. The calculation of the 


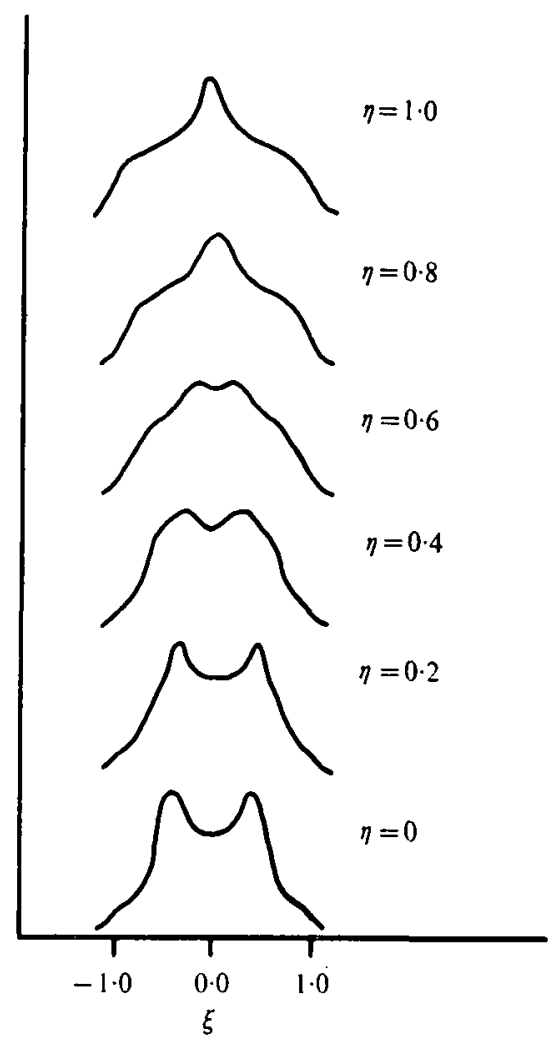

Fig. 6. Powder type spectra. Influence of the asymmetry parameter $\eta$. The curves were calculated assuming a Gaussian lineshape with constant linewidth $\sigma=0.1$ (Chiba (I962), $\mathcal{F}$. Chem. Phys.; reprinted with permission from the copyright owner.)

powder pattern is more complicated if the asymmetry parameter differs from zero and details may be found in Cohen \& Reif (1957). Computer spectra (Chiba, 1962) for various values of $\eta$ are shown in Fig. 6. By comparing Figs. 5 and 6 it can be seen that a non-zero asymmetry parameter or a large dipolar splitting may lead to quite similar line shapes for crystalline powder-type spectra.

B. Deuterium magnetic resonance of liquid crystals

\section{Anisotropic motions in liquid crystals}

Liquid crystals are fluid systems which at the same time exhibit a rather regular ordering of their constituent molecules. Depending on the chemical composition liquid crystals are classified as thermotropic or 
A

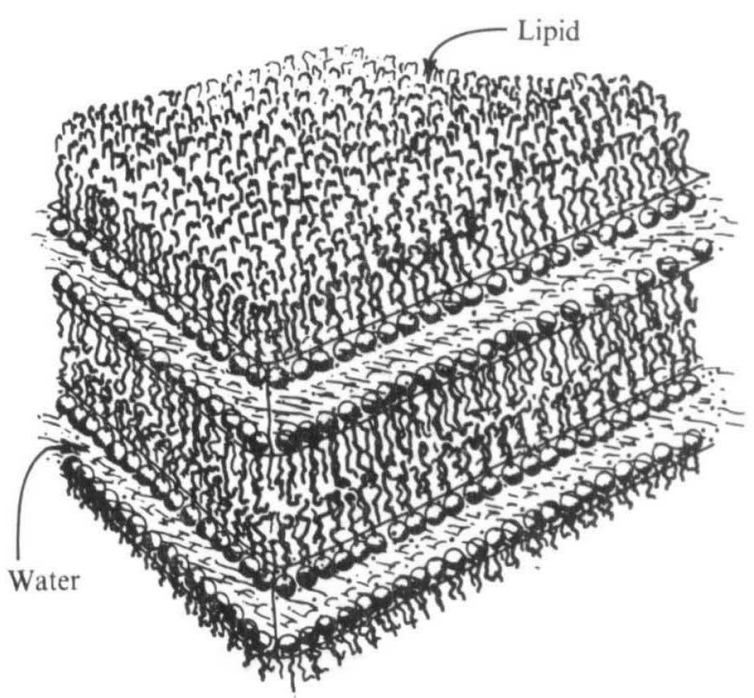

B

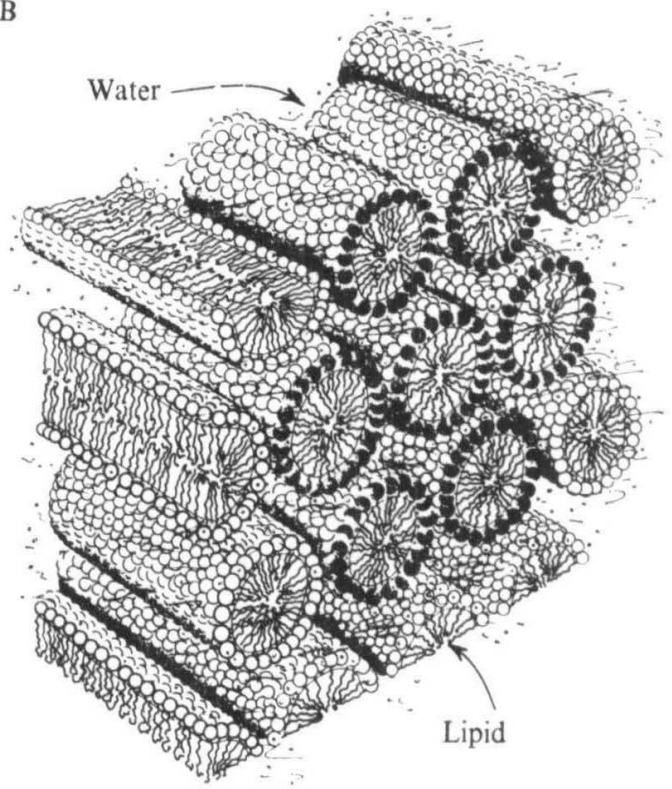

Fig. 7. Lyotropic liquid crystals. (A) Bilayer (lyotropic smectic phase); (B) cylinders (lyotropic hexagonal phase). (Reprinted from Rosevear (1968) F. Soc. Cosmetic Chem., with permission from the copyright owners.) 
lyotropic liquid crystals and both classes are further subdivided into a number of mesophases with different symmetries. The most important lyotropic mesophases are the lamellar and the hexagonal phases which are shown in Fig. 7. For a detailed description of the physical and chemical properties of liquid crystals the reader is referred to the books of Brown, Doane \& Neff (1971) and Gray \& Winsor (1974).

Liquid crystalline phases are characterized by their tendency to align the constituent rod-like molecules parallel to the long molecular axes. Due to the parallel packing, rotations perpendicular to the long axis are restricted while those around this axis are not hindered and occur with frequencies in the range of $10^{7}-10^{10} \mathrm{cps}$. The parallel ordering is, however, far from being perfect since angular excursions perpendicular to the long molecular axis are still possible. These oscillations are almost as rapid as the rotations around the long axis, however, they are confined to rather small angular amplitudes. The problem of how to define the average order in the system can then be approached in the following way. A macroscopic sample of liquid crystals is considered to be composed of a large number of microdomains. The average orientation of the ensemble of molecules (perhaps $10^{4}-10^{5}$ ) grouped together in one microdomain is characterized by a director $\mathbf{z}^{\prime}$. (In a lipid bilayer, for example, the director $\mathbf{z}^{\prime}$ at any point of the surface is identical with the normal on the bilayer surface.) Around this director axis the movements of the molecules are assumed to be cylindrically symmetric. The optical properties of such a microdomain are thus similar to a uniaxial crystal with $\mathbf{z}^{\prime}$ as the optical axis. The directions of the microregions will not necessarily be uniform in a macroscopic sample but we postpone a discussion of this effect for the moment and consider just one homogeneous microregion. Let us attach a cartesian coordinate system $\mathbf{x}, \mathbf{y}, \mathbf{z}$ to the liquid-crystal molecule and let us ask for the average fluctuations of these axes around the director axis $\mathbf{z}^{\prime}$. A convenient measure of the fluctuations is the so-called order parameter $S_{i i}$, which is defined as:

$$
S_{i i}=(\mathrm{I} / 2)\left(3 \overline{\cos ^{2} \theta_{i}}-1\right) \quad(i=1,2,3=x, y, z) .
$$

Here $\overline{\cos ^{2} \theta_{i}}$ denotes the time average of the angular fluctuations of the $i$ th coordinate axis with respect to the director axis $z^{\prime}$. From the orthogonality relation of the cosines

$$
\sum_{i=1}^{3} \cos ^{2} \theta_{i}=\mathrm{I}
$$


it immediately follows

$$
\sum_{i=1}^{3} S_{i i}=0
$$

Only two-order parameters are independent. Furthermore, since $\cos ^{2} \theta_{i}$ assumes values between $O$ and $I$ it also follows

$$
-\frac{1}{2} \leqslant S_{i i} \leqslant \mathrm{I} \text {. }
$$

Strictly speaking the order parameters $S_{i i}$ are the diagonal elements of a $3 \times 3$ ordering matrix which has been introduced to describe fluctuations of second-rank tensors in a most general manner (Saupe, 1964). This ordering matrix is symmetric $\left(S_{i j}=S_{j i}\right)$ and may contain up to five independent elements $S_{i j}$. It can be simplified if the tensor under investigation is given in the diagonal form under which conditions the average fluctuations are specified unequivocally by the order parameters $S_{i i}$ of the three principal axes. If the molecule fixed tensor is furthermore axially symmetric, say, with the z-axis as the symmetry axis, then it suffices to specify the order parameter $S_{33}$ of this axis. The other two axes $(\mathbf{x}, \mathbf{y})$ are physically indistinguishable and have the same order parameter $S_{11}=S_{22}$. From equation (40) it then follows

$$
S_{33}=-\frac{1}{2} S_{11}=-\frac{1}{2} S_{22} \text {. }
$$

The type of tensors which are experimentally accessible are the polarizability tensor $\left(\alpha_{i k}\right)$, the tensor of the dielectric constant $\left(\epsilon_{i k}\right)$, the electric field gradient tensor $\left(V_{i k}\right)$ (at the position of a certain nucleus), the dipolar interaction tensor $\left(D_{i j}\right)$ (magnetic interaction of two atomic nuclei), the chemical shielding tensor $\left(\sigma_{i j}\right)$, or analogous spectroscopic tensors. The orientation and anisotropies of these tensors depend strongly on the chemical structure of the molecule. Their principal values and the direction of the principal axes with respect to the molecular geometry must be inferred from single crystal measurements. Due to the fluctuations in a liquid crystal these tensor anisotropies will be partially averaged out. Comparing the remaining anisotropies with the single crystal data leads to a determination of the mean angular fluctuations $\overline{\cos ^{2} \theta_{i}}$ and the order parameters $S_{i i}$.

For most molecules the principal axes of the various tensors listed above will not coincide. Therefore order parameters determined with different experimental techniques will generally refer to different coordinate systems. In order to compare the data it is necessary to express them in the same molecular coordinate system. This new coordinate 
system can be chosen at will but a rather natural choice would be the principal axes system of the rotational diffusion tensor. The transformation itself is a geometric rotation of the ordering matrix, with the rotation angles being defined by the angles between the two sets of principal axes.

\section{Deuterium order parameters in planar-oriented liquid crystals}

Let us consider a liquid crystalline microdomain where at an arbitrary position in the constituent molecules a hydrogen has been replaced by deuterium. Since the molecular movements are anisotropic, the electric field gradient at the position of the deuterium nucleus is not completely averaged to zero and therefore gives rise to a quadrupole splitting. The magnitude of this splitting is easily derived by analogy with single crystals. For a single crystal the angular dependence of the quadrupole splitting was found to be

$$
\Delta \nu_{Q}(\theta, \phi)=\frac{3}{2}\left(\frac{e^{2} q Q}{h}\right)\left(\frac{3 \cos ^{2} \theta-1}{2}+\frac{1}{2} \eta \sin ^{2} \theta \cos 2 \phi\right) .
$$

Here $\theta$ and $\phi$ are the Eulerian angles defining the orientation of the electric field gradient tensor with respect to the magnetic field $\mathbf{H}_{0}$. The unique axis of a liquid crystal is the director axis $z^{\prime}$. If the magnetic field is applied parallel to this axis, the instantaneous position of the field gradient tensor is specified unambiguously by the same angles $\theta$ and $\phi$ as defined above. The additional feature of the liquid crystal then is the rapid fluctuations of the molecules around the director axis. The static field gradient tensor is averaged by this motion and the new effective field gradient tensor is axially symmetric with respect to $\mathbf{z}^{\prime}$. Since the rate of the fluctuations is much faster than the time scale of the deuterium nmr experiment, only this average field gradient is detected. This means that the angular functions in equation (23) must be replaced by their time average:

$$
\Delta v_{Q}(\theta, \phi)=\frac{3}{2}\left(\frac{e^{2} q Q}{h}\right)\left(\frac{3 \overline{\cos ^{2} \theta}-\mathrm{I}}{2}+\frac{1}{2} \eta \overline{\sin ^{2} \theta \cos 2 \phi}\right) .
$$

Now let $\mathbf{x}, \mathbf{y}$, and $\mathbf{z}$ be the unit vectors along the principal axes of the static electric field gradient tensor. The direction cosines of these axes with respect to the director axis $\mathbf{z}^{\prime}$ are then given by

$$
\begin{aligned}
& \mathbf{x} \mathbf{z}^{\prime}=\cos \theta_{1}=\sin \theta \cos \phi, \\
& \mathbf{y} \mathbf{z}^{\prime}=\cos \theta_{2}=\sin \theta \sin \phi, \\
& \mathbf{z} \mathbf{z}^{\prime}=\cos \theta_{3}=\cos \theta
\end{aligned}
$$


Using these relations and taking into account the definition of order parameters according to (39), equation (4I) may be rewritten as

$$
\Delta \nu_{Q}(\theta, \phi)=\frac{3}{2}\left(\frac{e^{2} q Q}{h}\right)\left(S_{33}+\frac{1}{3} \eta\left[S_{11}-S_{22}\right]\right) .
$$

In the most general case the quadrupole splitting of a singly deuterated liquid crystal is therefore determined by two order parameters (the third is fixed due to $\sum_{i=l}^{3} S_{i i}=0$ ). This means that for $\eta \neq 0$ one-order parameter must be known before the other can be evaluated from the observed deuterium quadrupole splitting. Quite often, however, the asymmetry parameter is small and can be neglected. For $\mathrm{C}-\mathrm{D}$ bonds, for example, it is known from theoretical calculations (Hoyland, 1968) as well as crystal studies (Derbyshire, Gorvin \& Warner, 1969; Barnes \& Bloom, 1973; cf. also Fung \& Wei, 1970; Fung, 1974) that the asymmetry parameter is $\eta \lesssim 0.05$. Neglecting the asymmetry parameter leads to

$$
\Delta \nu_{Q}(\theta)=\frac{3}{2}\left(\frac{e^{2} q Q}{h}\right) S_{33}
$$

and the quadrupole splitting depends on one order parameter only. If the magnetic field $\mathbf{H}_{0}$ is not parallel to the director $\mathbf{z}^{\prime}$ but makes an angle $\beta^{\prime}$ with this axis the observed quadrupole splitting is

$$
\Delta \nu_{Q}\left(\theta, \beta^{\prime}\right)=\frac{3}{2}\left(\frac{e^{2} q Q}{h}\right) S_{33}\left(\frac{3 \cos ^{2} \beta^{\prime}-1}{2}\right) .
$$

The quadrupole splitting collapses if the director axis $z^{\prime}$ is inclined at the 'magic angle' $\left(\beta^{\prime}=54^{\circ} 74^{\circ}\right)$ with respect to $\mathbf{H}_{0}$.

Equation (45) is readily derived by means of the irreducible tensor representation. Using Wigner rotation matrices the two consecutive rotations can be written as

$$
V_{H}^{(2, r)}=\sum_{q} D_{q r}^{(2)}\left(\alpha^{\prime} \beta^{\prime} \gamma^{\prime}\right) \sum_{p} D_{p q}^{(2)}(\alpha \beta \gamma) V_{P}^{(2, p)}
$$

The first transformation (Eulerian angles $\alpha, \beta, \gamma$ ) rotates the field gradient tensor $\mathbf{V}_{P}$ from its molecule fixed principal coordinate system into the coordinate system of the director $\mathbf{z}^{\prime}$, the second transformation (Eulerian angles $\left.\alpha^{\prime} \beta^{\prime} \gamma^{\prime}\right)$ rotates the director coordinate system into the laboratory coordinate system defined by the direction of the external magnetic field. The rotation around the director axis $\mathbf{z}^{\prime}$ is specified by the angle $\gamma$. Taking into account the cylindrical symmetry of the system around $z^{\prime}$ all matrix elements containing $\gamma$ are averaged to zero yielding 


$$
\begin{aligned}
& \left.\overline{V_{H}^{(2,0)}}=D_{00}^{(2)}\left(\beta^{\prime}\right) \sum_{p} \overline{D_{p 0}^{(2)}(\alpha \beta}\right) V_{P}^{(2, p)} \\
& \overline{V_{H}^{(2,0)}}=\left(\frac{3 \cos ^{2} \beta^{\prime}-1}{2}\right)\left(\overline{\frac{3 \cos ^{2} \beta-1}{2}} V_{z z}+\frac{1}{2} \overline{\sin ^{2} \beta \cos 2 \alpha}\left[V_{x x}-V_{y y}\right]\right) .
\end{aligned}
$$

Setting $\alpha=\phi$ and $\beta=\theta$ this may be written as

$$
\overline{V_{H}^{(2,0)}}=\left(\frac{3 \cos ^{2} \beta^{\prime}-1}{2}\right) V_{z z}\left(S_{33}+\frac{1}{3} \eta\left[S_{11}-S_{22}\right]\right) .
$$

Insertion into equation (20) yields

$\Delta \nu_{Q}\left(\theta, \phi, \beta^{\prime}\right)=\Delta v_{Q}\left(S_{i i}, \beta^{\prime}\right)=\left(\frac{3}{2}\right)\left(\frac{e^{2} q Q}{h}\right)\left(S_{33}+\frac{1}{3} \eta\left[S_{11}-S_{22}\right]\right)\left(\frac{3 \cos ^{2} \beta^{\prime}-1}{2}\right)$,

which for $\eta=0$ reduces to equation (45).

The above formalism will be illustrated for a deuterated lipid bilayer (Seelig \& Niederberger, 1974 $a$ ). The bilayer investigated here is a lyotropic liquid crystal composed of a fatty acid, a deuterated long chain alcohol and water (phase composition: sodium octanoate $34 \mathrm{wt} . \%, \mathrm{r}, \mathrm{r}$-dideuteriooctanol $30 \mathrm{wt} . \%$, water $36 \mathrm{wt} . \%$ ). X-ray diffraction shows that in this ternary mixture the lipid molecules are arranged in bilayers which are separated from each other by layers of water. Both amphiphilic components, the fatty acid as well as the alcohol, are integral constituents of the lipid region. A macroscopic ordering of this phase can be induced by forcing it between two closely spaced, parallel glass surfaces. Shearing forces and the contact with the glass surfaces arrange the microdomains in parallel layers on the supporting plates. The director axes $\mathbf{z}^{\prime}$ are parallel to each other and point perpendicular to the glass surfaces. The normal to the glass plates is the optical axis of the liquid crystalline system (Seelig, r 970 ). Figure 8 shows the deuterium nmr spectra of such oriented bilayers. The two deuterons in I, I-dideuteriooctanol behave in a motionally equivalent manner and give rise to the same quadrupole splitting. $\beta^{\prime}$ denotes the angle between the magnetic field and the normal of the glass plates. The angular dependence of the quadrupole splitting obeys equation (45), in particular it collapses at the 'magic angle'. Extrapolation of the observed quadrupole splittings to $\beta^{\prime}=0^{\circ}$ leads to a maximum splitting of $\Delta v_{Q}=56 \cdot 6 \pm 0.6 \mathrm{kHz}$. Since the quadrupole splitting constant $\left(e^{2} q Q / h\right)$ for $\mathrm{C}-\mathrm{D}$ bonds of paraffins has been found to 

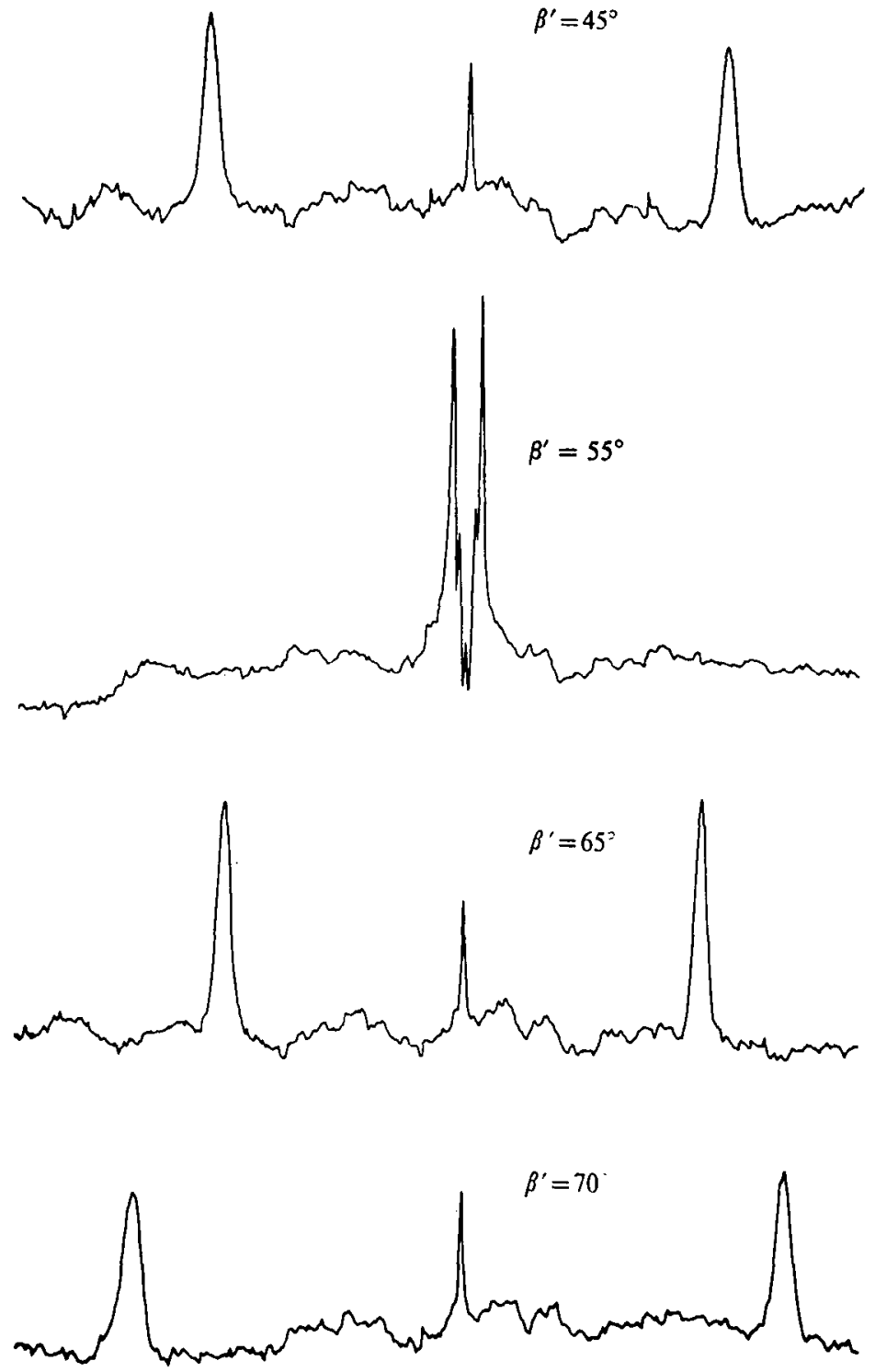

$10 \mathrm{kHz}$

Fig. 8. Deuterium nmr spectra $(13.8 \mathrm{MHz})$ of oriented bilayers. Phase composition: sodium octanoate $34 \mathrm{wt} . \%, 1,1$-dideuteriooctanol $30 \mathrm{wt}$. \%, and water $36 \mathrm{wt}$. $\%$. Approximately $20 \mathrm{mg}$ liquid crystalline phase was sandwiched between a stack of 20 glass plates. $\beta^{\prime}$ denotes the angle between the magnetic field and the normal to the glass plates which is also the normal to the oriented bilayers. The sharp signal in the centre arises from isotropic motion of deuterium labelled molecules. During the preparation of the oriented sample a small fraction of an isotropic phase seems to form. 
be $170 \mathrm{kHz}$ (Burnett \& Muller, 197I) the order parameter $S_{33}$ is readily evaluated from equation (45). In the case of $\mathrm{C}-\mathrm{D}$ bonds the dominant axis of the axially symmetric field gradient tensor $V_{P}$ is identical with the C-D bond vector. Therefore $S_{33}$ may also be called $S_{\mathrm{CD}}$ and can be visualized as a measure for the angular excursions of the $\mathrm{C}-\mathrm{D}$ bond vector around the director axis $\mathbf{z}^{\prime}$. Experimentally, only the absolute value of the quadrupole splitting is accessible (unless $S_{\mathrm{C}-\mathrm{D}}>0.5$, in which case $\Delta \nu_{Q}$ is positive). For a lipid bilayer the sign of $\Delta \nu_{Q}$ can sometimes be deduced from geometrical considerations. For example, in the hydrocarbon region the long molecular axis of the lipid molecule is essentially parallel to the axis of motional averaging, and the $\mathrm{C}-\mathrm{D}$ bond of a methylene unit is then oriented perpendicular to this axis. Such an orientation requires negative order parameters and for the example shown in Fig. 8 is thus follows $S_{\mathrm{CD}}=-0.22$.

\section{Line shapes for random and cylindrical distributions of liquid crystalline microdomains}

Random distribution. In many experimental situations a random distribution of the director axes $\mathbf{z}^{\prime}$ of liquid crystalline microdomains is encountered. The line shape given by such a sample can be derived in close analogy with the polycrystalline powder pattern ( $\left(I I, A_{5}\right.$ ), the only difference being that the resonance lines depend now on the orientation of the director axis $z^{\prime}$ instead of the orientation of the nuclear site. Combining equations (19) and (48) the angular dependence of the resonance positions $\nu_{ \pm}$for one microdomain is given by

$$
h \nu_{ \pm}=g \beta_{N} H_{0} \pm \frac{3}{4}\left(e^{2} q Q\right)\left(S_{33}+\frac{1}{3} \eta\left[S_{11}-S_{22}\right]\right)\left(\frac{3 \cos ^{2} \beta^{\prime}-1}{2}\right) .
$$

$\beta^{\prime}$ is the angle between the director axis and the magnetic field. For a random distribution of director axes the probability density $p\left(\beta^{\prime}\right)$ is $p\left(\beta^{\prime}\right)=\frac{1}{2} \sin \beta^{\prime}$. We define a reduced resonance frequency $\xi_{ \pm}$according to

$$
\xi_{ \pm}= \pm \frac{\nu_{ \pm}-\left(g \beta_{N} H_{0} / h\right)}{(3 / 4)\left(e^{2} q Q / h\right)\left(S_{33}+\frac{1}{3} \eta\left[S_{11}-S_{22}\right]\right)}=\frac{3 \cos ^{2} \beta^{\prime}-1}{2}
$$

Averaging over all angles $\beta^{\prime}$ leads to the theoretical powder pattern shown in Fig. 3. The separation of the two most intense peaks is given by

$$
\begin{aligned}
& \Delta \xi_{\text {powder }}=\text { I or } \\
& \Delta \nu_{\text {powder }}=\frac{3}{4}\left(e^{2} q Q / h\right)\left(S_{33}+\frac{1}{3} \eta\left[S_{11}-S_{22}\right]\right) .
\end{aligned}
$$

A good example for a random distribution of microdomains is a coarse dispersion of phospholipid bilayers in water (Stockton et al. 1974; Gally 


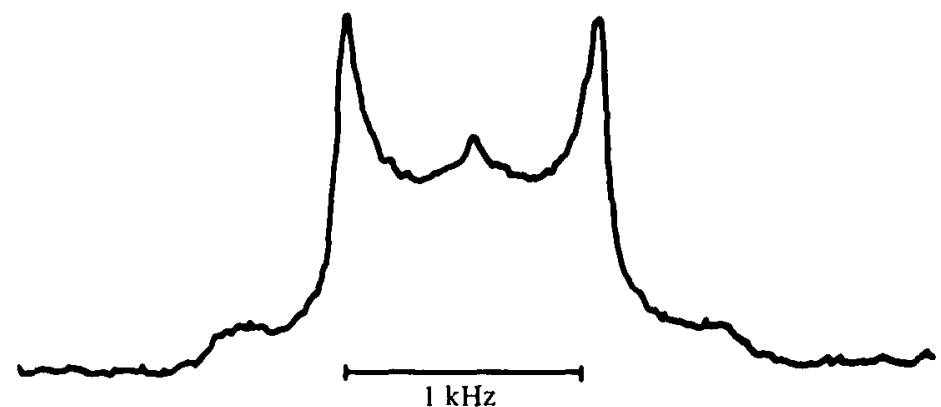

Fig. 9. Deuterium magnetic resonance spectrum (13.8 MHz) of bilayers of dipalmitoyl-3-sn-phosphatidylcholine deuterated at the N-CD, group $\left(\mathrm{NCD}_{3}\left(\mathrm{CH}_{3}\right)_{2}\right.$-DPL). Phase composition: $51 \cdot 5 \% \mathrm{H}_{2} \mathrm{O}, 48.5 \%$ DPL. Temperature $=59^{\circ} \mathrm{C}$. (Reprinted from Gally, Niederberger \& Seelig (1975), Biochemistry, with permission from the copyright owner.)

et al. 1975). Fig. 9 shows the spectrum of a dispersion of dipalmitoyllecithin deuterated at the $N$-methyl group. The spectrum has clearly the shape predicted by Fig. 5. The peak-to-peak separation is $\Delta v_{\text {powder }}=$ I.06 kHz. Taking into account the linewidth contributions a computer fit of this spectrum shows that the true separation is $\Delta \nu_{\text {powder }} \approx \mathrm{I} \cdot \mathrm{I} 6 \mathrm{kHz}$ (Seelig et al. 1977).

It should be noted that the asymmetry parameter $\eta$ influences only the peak-to-peak separation but not the shape of the spectrum. This difference compared to crystals arises from the fact that the effective (timeaveraged) electric field gradient tensor $\mathbf{V}$ in the liquid crystal is always axially symmetric around $\mathbf{z}^{\prime}$.

Oriented cylinders: no lateral diffusion. Next we consider a sample of cylindrical liquid crystals (Fig. $7 \mathrm{~B}$ ) in which the cylinder axes $\mathbf{z}$ are aligned parallel to each other. The constituent molecules are distributed evenly in planes perpendicular to the $z$-axis. We assume that during the time of the measurement the molecules are rapidly rotating and fluctuating around their long molecular axis but remain located at their lattice site (no lateral diffusion). This (hypothetical) situation corresponds to a radial distribution of director axis $\mathbf{z}^{\prime}$ around the cylinder axis $\mathbf{z}$. The geometry of this system is shown in Fig. IO. According to this figure the direction of an individual director $\mathbf{z}^{\prime}$ is given by

$$
\mathbf{z}^{\prime}=(\cos \phi, \sin \phi, o)
$$

and the probability density of $\phi$ is

$$
p(\phi)=\frac{1}{2 \pi} .
$$


Deuterium magnetic resonance

379

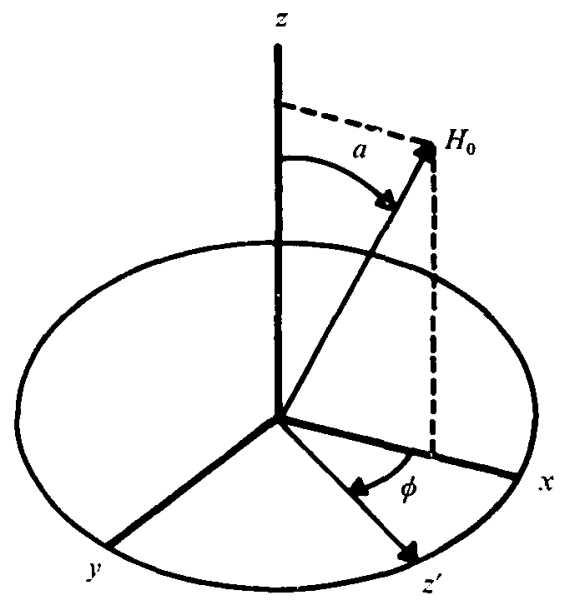

Fig. Io. Cylindrical distribution of director axes $z^{\prime}$. The cylinder axis is given by $z$. The magnetic field $H_{0}$ is inclined at an angle $\alpha$ with respect to $z$.

If the external magnetic field $\mathbf{H}_{0}$ is inclined at an angle $\alpha$ with respect to the cylinder axis its orientation in the $\mathbf{x}, \mathbf{y}, \mathbf{z}$-coordinate system is given by

$$
\mathbf{h}=(\sin \alpha, 0, \cos \alpha) .
$$

The direction cosine between the magnetic field and a director $\mathbf{z}^{\prime}$ is thus

$$
\cos \beta^{\prime}=\mathbf{h} \mathbf{z}^{\prime}=\sin \alpha \cos \phi .
$$

According to equation (5I) the angular dependence of the (reduced) resonance positions is then found to be

$$
\xi_{ \pm}= \pm \frac{3 \sin ^{2} \alpha \cos ^{2} \phi-I}{2} .
$$

Since

$$
p\left(\xi_{ \pm}\right)=p(\phi) \frac{\mathrm{d} \phi}{\mathrm{d} \xi_{ \pm}}=\frac{\mathrm{I}}{2 \pi} \frac{\mathrm{d} \phi}{\mathrm{d} \xi_{ \pm}} .
$$

This leads to the following expression for the probability density of the resonance frequencies.

$$
p\left(\xi_{ \pm}\right) \propto\left[\left( \pm 2 \xi_{ \pm}+\mathrm{I}\right)\left(3 \sin ^{2} \alpha \mp 2 \xi_{ \pm}-\mathrm{I}\right)\right]^{-\frac{1}{2}} .
$$

For $\alpha=0^{\circ}$ (magnetic field parallel to cylinder axis $z$ ) two sharp resonance lines with a separation of $\Delta \xi=\mathrm{r}$ or

$$
\Delta \nu_{Q}=\left(\frac{3}{4}\right)\left(e^{2} q Q / h\right)\left(S_{33}+\frac{1}{3} \eta\left[S_{11}-S_{22}\right]\right)
$$

Downloaded from https://www.cambridge.org/core. WWZ Bibliothek, on 14 Nov 2017 at 10:27:10, subject to the Cambridge Core

terms of use, available at https://www.cambridge.org/core/terms. https://doi.org/10.1017/S0033583500002948 

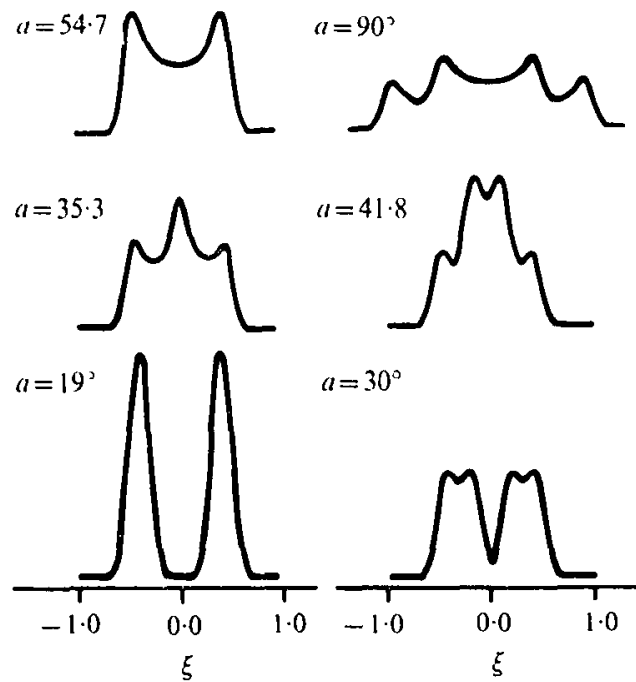

$a=30^{\circ}$

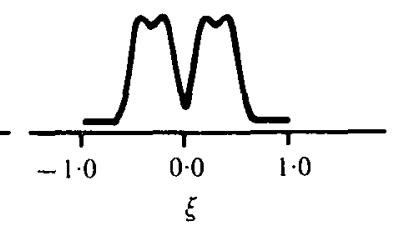

Fig. Ir. Theoretical spectra for a cylindrical distribution of director axes. The spectra were calculated using Gaussian lineshapes with a constant linewidth $\sigma=0.07 . \alpha$ is the angle between the magnetic field and the cylindrical symmetry axis.

are observed. For an arbitrary orientation $\alpha$ equation (56) predicts a superposition of lines with singularities at $\xi= \pm \frac{1}{2}$ and

$$
\xi_{ \pm}= \pm(1 / 2)\left(3 \sin ^{2} \alpha-1\right) \text {. }
$$

Thus four intense peaks are expected with separations of

and

$$
\Delta v_{Q}=(3 / 4)\left(e^{2} q Q / h\right)\left(S_{33}+\frac{1}{3} \eta\left[S_{11}-S_{22}\right]\right)
$$

$$
\Delta v_{Q}=(3 / 4)\left(e^{2} q Q / h\right)\left(S_{33}+\frac{1}{3} \eta\left[S_{11}-S_{22}\right]\right)\left(3 \sin ^{2} \alpha-\mathrm{I}\right) .
$$

Theoretical spectra for various orientation angles $\alpha$ are shown in Fig. I I.

Oriented cylinders. Rotation around the cylinder axis. A different picture emerges if the liquid crystal molecules rotate rapidly around the cylinder axis or if the cylinder itself rotates around its long axis. This leads to an additional averaging effect around the cylinder axis. The angular dependence of the (reduced) resonance frequencies is derived from equation (54)

$$
\xi_{ \pm}= \pm \frac{3 \sin ^{2} \alpha \overline{\cos ^{2} \phi}-I}{2}
$$


where $\alpha$ is again the angle between the magnetic field $H_{0}$ and the cylinder axis. Since

it follows

$$
\overline{\cos ^{2} \phi}=\frac{1}{2 \pi} \int_{0}^{2 \pi} \cos ^{2} \phi \mathrm{d} \phi=\frac{1}{2}
$$

$$
\xi_{ \pm}= \pm \frac{(3 / 2) \sin ^{2} \alpha-1}{2}=\mp \frac{3 \cos ^{2} \alpha-1}{4} \text {. }
$$

For all orientations $\alpha$ only two sharp resonance lines are observed with a frequency separation of $\Delta \xi=\frac{1}{2}\left(3 \cos ^{2} \alpha-1\right)$

or

$$
\Delta \nu_{Q}=(3 / 4)\left(e^{2} q Q / h\right)\left(S_{33}+\frac{1}{3} \eta\left[S_{11}-S_{22}\right]\left(\frac{3 \cos ^{2} \alpha-\mathrm{I}}{2}\right) .\right.
$$

Except for a factor $\frac{1}{2}$ this result is identical with equation (49) derived for planar oriented systems. Equation (62) describes the magnetic behaviour of one molecular cylinder. It is equally applicable to a macroscopic sample of liquid crystals if the cylinder axes are aligned parallel to each other.

Random distribution of cylinders. If the cylinders are distributed at random the resonance positions given by equation (6I) must be averaged over all orientations of the cylinder axes. The weighting function is $p(\alpha)=(1 / 2) \sin \alpha$ and the calculation of the line shape is performed as discussed for the crystalline powder. The result is the spectral shape shown in Fig. 3 with the frequency spacing of most intense peaks given by

$$
\Delta v_{Q}=(3 / 8)\left(e^{2} q Q / h\right)\left(S_{33}+\frac{1}{3} \eta\left[S_{11}-S_{22}\right]\right) .
$$

Compared to a random distribution of normal liquid crystals (equation (52)) the splitting is reduced by a factor of 2 due to the additional rotation around the cylinder axis.

Trofold cylindrical symmetry. The last type of geometry to be discussed in connexion with cylindrical liquid crystals is an alignment of cylinders in parallel planes with a random distribution of the cylinder axes within the planes. With respect to the normal to the planes the distribution of the cylinder axes is cylindrically symmetric. This distribution leads to the type of spectra shown in Fig. II, but the separation of the peaks is reduced by a factor 2 compared to the static case due to the rotation around the cylinder axis:

and

$$
\Delta v_{Q}=(3 / 8)\left(e^{2} q Q / h\right)\left(S_{33}+\frac{1}{3} \eta\left[S_{11}-S_{22}\right]\right)
$$




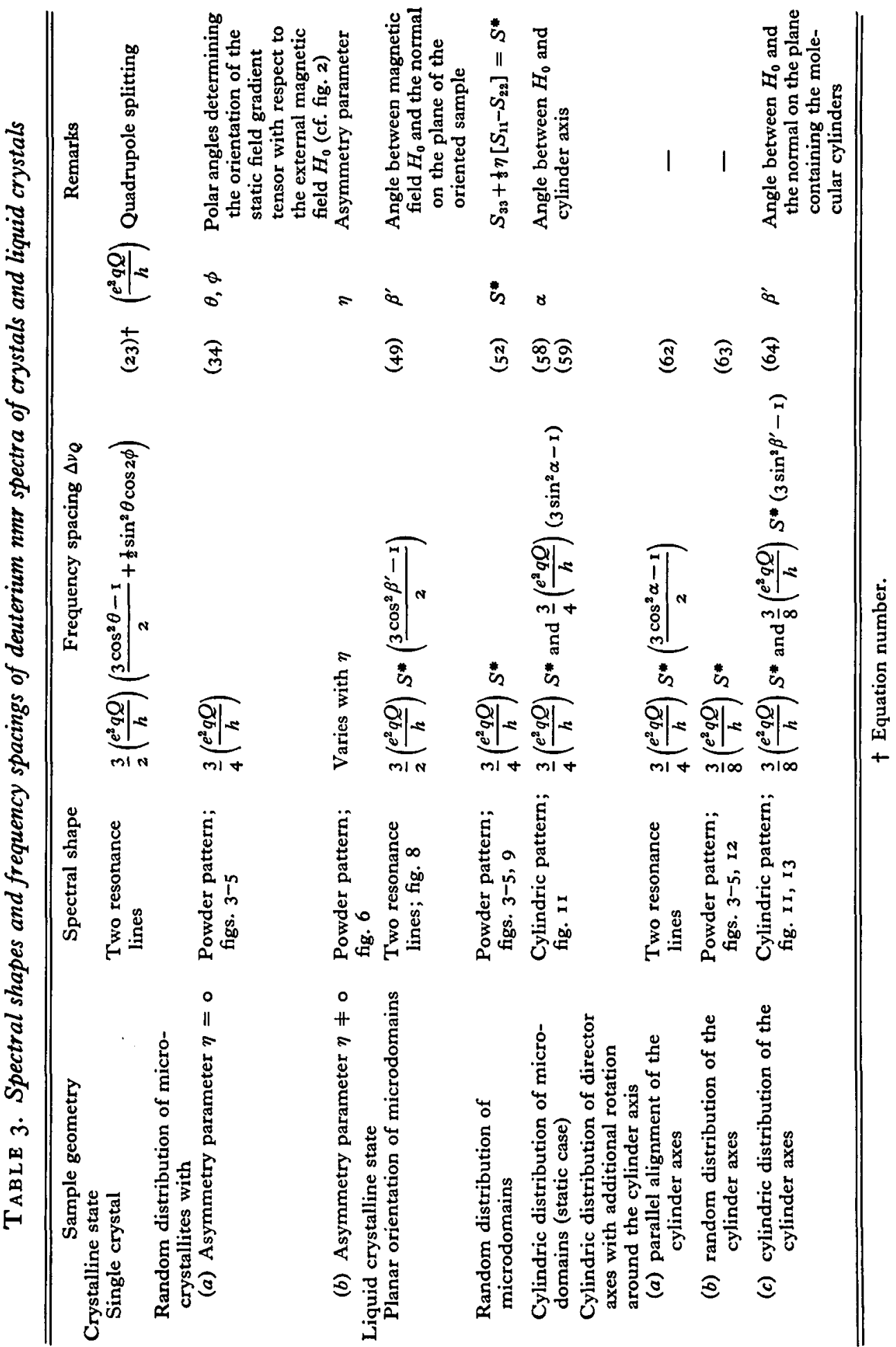




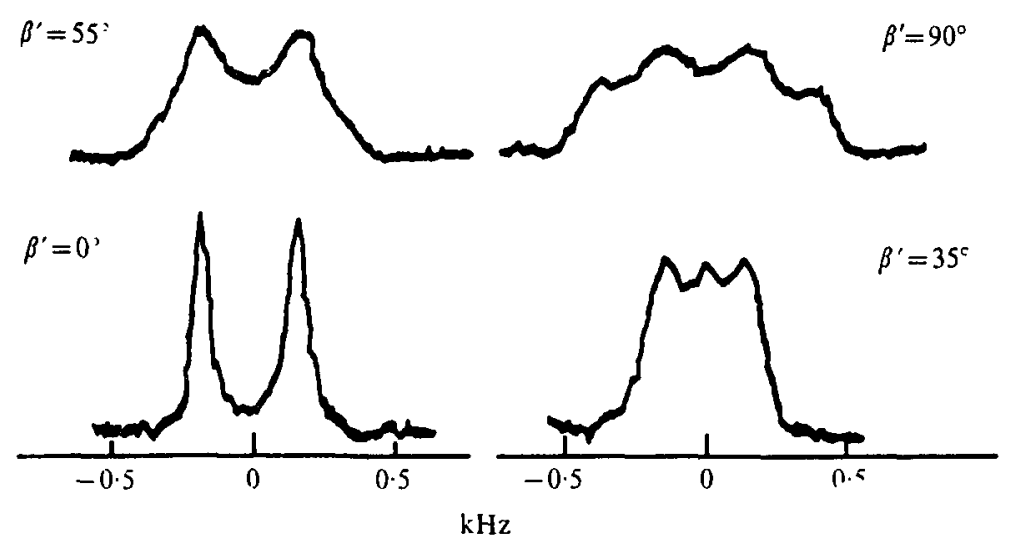

Fig. 12. Hexagonal liquid crystals oriented between glass plates (potassium laurate 40 wt. $\%, \mathrm{D}_{2} \mathrm{O} 60 \mathrm{wt} . \%$ ) at $50^{\circ} \mathrm{C} . \beta^{\prime}$ is the angle between the magnetic field and the normal to the supporting plates (Mely, Charvolin \& Keller, Chem. Phys. Lip. (1975), reprinted with permission from the copyright owner).

Here $\beta^{\prime}$ denotes the angle between the external magnetic field and the normal to the plane containing the molecular cylinders.

The different geometries and motional states together with the corresponding line shapes and frequency spacings are summarized in Table 3 . A suitable experimental system exhibiting cylindrical symmetry is the hexagonal phase shown in Fig. 7 B. In this lyotropic liquid crystal the amphiphilic molecules are arranged in rods of cylindrical cross-section which are surrounded by water. A practical example for a hexagonal phase is a mixture of potassium laurate (40 wt. \%) and $\mathrm{D}_{2} \mathrm{O}(60 \mathrm{wt} . \%)$ at $35-70^{\circ} \mathrm{C}$ (Mely et al. 1975). In this system the $\mathrm{D}_{2} \mathrm{O}$ molecules are partially ordered on the surface of the cylinders. If this phase is pressed between glass plates, then the deuterium spectra follow the pattern predicted by equation (64) and Fig. II, as is illustrated in Fig. I2. This indicates that the cylinder axes are now aligned parallel to the glass surface but that there exists no preferential orientation within the plane.

\section{Anisotropic rotation of $C D_{2}$ and $C D_{3}$ groups}

So far we have been concerned with molecules labelled with one deuteron only. Next we consider the motion of a $\mathrm{CD}_{2}$ segment carrying two deuterons. For the sake of simplicity we assume a homogeneously orientated liquid crystal or membrane with the orientation specified by the director axis $\mathbf{z}^{\prime}$. Both deuterons then rotate around $\mathbf{z}^{\prime}$ and since the asymmetry 
parameter $\eta$ of a $\mathrm{C}-\mathrm{D}$ bond is practically zero, the effective quadrupole splitting is given by (cf. equation 44)

$$
\Delta v_{Q}(\theta)=(3 / 2)(e Q / h) \overline{\left.V^{(2,} 0\right)}=(3 / 2)\left(e^{2} q Q / h\right) S_{\mathrm{CD}} .
$$

$A$ priori the movements and hence the order parameters of the two $\mathrm{C}-\mathrm{D}$ bond vectors cannot be expected to be identical. Consider for example the extreme situation where one $\mathrm{C}-\mathrm{D}$ bond is aligned parallel to the director axis $\mathbf{z}^{\prime}$ and constitutes the rotation axis for the other $\mathrm{C}-\mathrm{D}$ bond vector. The order parameters for the $C-D(I)$ and the $C-D(2)$ bond are then

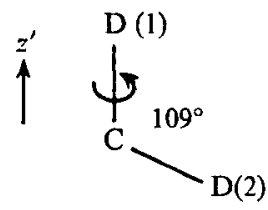

I and $\left(3 \cos ^{2} 109-I\right) / 2=-(\mathrm{I} / 3)$, respectively, and two different quadrupole splittings are observed. However, in most liquid crystals and lipid bilayers investigated to date only one quadrupole splitting has been observed. In these systems the two deuterons of a $\mathrm{CD}_{2}$ group are therefore equivalent and are characterized by the same order parameter.

Unfortunately, the knowledge of the $\mathrm{C}-\mathrm{D}$ splittings is not sufficient in order to allow a quantitative description of the movement of the $\mathrm{CD}_{2}$ segment as a whole. This can be seen by transforming the electric field gradients of the two deuterons into a segment-fixed $\xi, \rho, \zeta$ coordinate system as is shown in Fig. I3. If the two deuterons are rotated around the $\rho$ axis by an angle $\pm \beta$, the irreducible tensor components in the new coordinate system (subscript $s$ ) are given by

$$
\begin{aligned}
& V_{s}^{(2,0)}=(\mathrm{I} / 2)\left(3 \cos ^{2}( \pm \beta)-\mathrm{I}\right) e q, \\
& V_{s}^{(2, \pm 1)}= \pm \sqrt{\frac{3}{2}} \sin ( \pm \beta) \cos ( \pm \beta) e q, \\
& V_{8}^{(2, \pm 2)}=\sqrt{\frac{3}{8}} \sin ^{2}( \pm \beta) e q .
\end{aligned}
$$

In a second transformation (Eulerian angles $\alpha^{\prime}, \beta^{\prime}, \gamma^{\prime}$ ) the segment-fixed tensor is referred to the coordinate system of the director axis $\mathbf{z}^{\prime}$ (subscript $H$ ). By invoking the rotational symmetry around $\mathbf{z}^{\prime}$ we find

$$
\begin{aligned}
\overline{V_{H}^{(2,0)}( \pm \beta)} & =e q\left\{\left(\frac{3 \cos ^{2} \beta-1}{2}\right) \overline{\left(\frac{3 \cos ^{2} \beta^{\prime}}{2}\right)} \frac{1}{2}\right) \frac{3}{4} \sin 2 \beta \overline{\sin 2 \beta^{\prime} \cos \alpha^{\prime}} \\
& \left.+\frac{3}{4} \sin ^{2} \beta \overline{\sin ^{2} \beta^{\prime} \cos 2 \alpha^{\prime}}\right\} .
\end{aligned}
$$




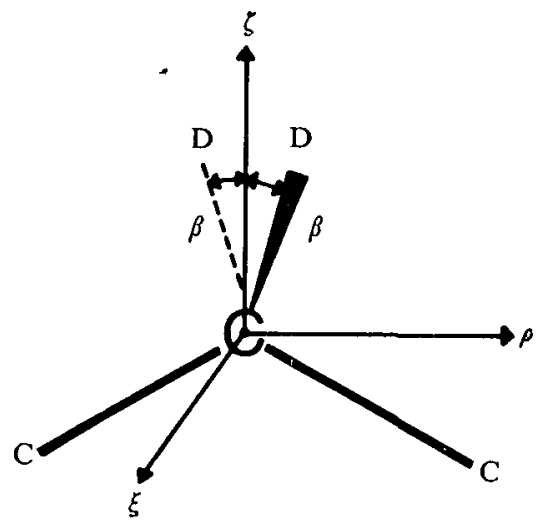

Fig. 13. Geometry of a $\mathrm{CD}_{2}$-group.

In the general case where two signals are observed the description of the movement of the $\mathrm{CD}_{2}$ segment requires the determination of three angular averages (equivalent to three independent order parameters). If the two deuterons give rise to the same quadrupole splitting the above expression reduces to

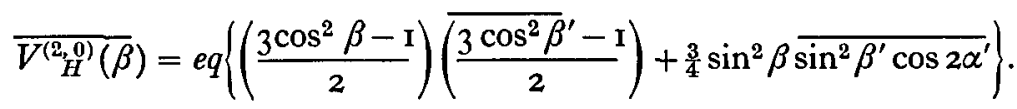

Only two angular averages (two-order parameters) must be specified and it can be concluded that the rotation axis of the segment lies either in the $\xi-\rho$ or in the $\rho-\zeta$ plane. However, in both situations the number of unknown parameters exceeds the number of experimental parameters and additional information from different types of experiments is required.

For hydrocarbon chains in lipid bilayers it has been assumed that the motion of the methylene groups is axially symmetric around the long molecular axis ( $\rho$-axis of Fig. 13) (Seelig \& Niederberger, 1974 $a, b$; Seelig \& Seelig, $1974 a, b)$. This is supported by proton decoupled deuterium nmr spectra of such systems (Diehl \& Niederberger, I974). Upon proton decoupling the deuterium resonance lines sharpen into a triplet. The remaining splittings are due to the deuteron-deuteron dipolar interactions (Fig. I4). From the direct deuteron-deuteron coupling the order parameter $\left|S_{\mathrm{DD}}\right|$ of the D-D axis can be derived. Close agreement was obtained between $\left|S_{\mathrm{DD}}\right|$ and the order parameter $\left|S_{\mathrm{CD}}\right|$ of the $C D$ axis which confirms the axial symmetry of the movement of 
the methylene segments in these membranes. For the case of an axially symmetric rotation around the $\rho$-axis the order parameter $S_{\rho}$ of this axis is related to $S_{\mathrm{CD}}$ according to

$$
S_{\rho}=\left(\frac{3 \cos ^{2} \beta^{*}-\mathrm{I}}{2}\right)^{-1} S_{\mathrm{CD}}
$$

where $\beta^{*}$ is the angle between the $\mathrm{C}-\mathrm{D}$ bond vector and the $\rho$-axis.

Close to the lipid-water interface the motion of the methylene segments may become dependent on the conformational state of the polar anchoring group. Higgs \& MacKay (1977) have determined the complete ordering tensor for the $\alpha$-methylene group of a potassium palmitatewater bilayer by measuring the quadrupole splitting of a selectively deuterated sample as well as the proton-proton dipolar splitting of the $\alpha$-segment. For the latter experiment perdeuterated potassium palmitate was selectively protonated in the $\alpha$-position. The two order parameters, $S_{\mathrm{CD}}$ and $S_{\mathrm{HH}}$, differ between 0-20\% in the temperature range studied $\left(40-100^{\circ} \mathrm{C}\right.$ ), indicating that the motion of this particular segment is not truly axially symmetric around the $\rho$-axis defined above.

The analysis of the motion of the $\mathrm{CD}_{3}$ group is straightforward. Since the $\mathrm{CD}_{3}$ group possesses a threefold symmetry axis, its motion is specified by the order parameter of this axis which can be deduced from $S_{\mathrm{CD}}$ by means of equation (68). For example from the quadrupole splitting of Fig. 9 the order parameter of the $\mathrm{C}-\mathrm{D}$ bond of the choline-methyl rotor is found to be $\left|S_{\mathrm{CD}}\right| \approx 0.009$ (equation $52 ; \eta=0 ; e^{2} q Q / h=170 \mathrm{kHz}$ ). Assuming tetrahedral bond angles the order parameter of the $\mathrm{N}-\mathrm{C}$ symmetry axis, $S_{\mathrm{NC}}$, is calculated to be

$$
\left|S_{\mathrm{NC}}=\right|\left(3 \cos ^{2} 109 \cdot 4-\mathrm{r}\right) /\left.2\right|^{-1} S_{\mathrm{CD}}=3 S_{\mathrm{CD}}
$$

\section{Deuterium quadrupole relaxation in anisotropic media}

The measurement of deuterium $T_{1}$ and $T_{2}$ relaxation times offers the advantage that the dominant relaxation pathway is quadrupole relaxation. This obviates some of the difficulties inherent to the interpretation of proton relaxation times where intra- and intermolecular mechanisms may contribute to the relaxation. A detailed discussion of deuterium relaxation theory is however beyond the scope of this article and only a few pertinent references can be given here. The simplest case of deuteron relaxation is that of a fast-tumbling sphere in an isotropic liquid. In the 

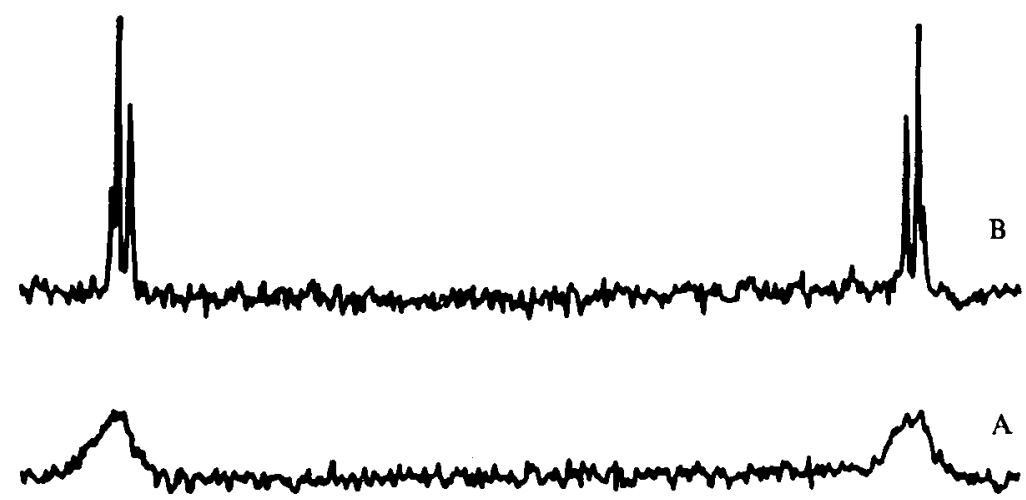

$1 \mathrm{kHz}$

Fig. 14. (A) Deuterium nmr spectrum of $n$-propanol-1, I $d_{2}$ r 5 mole $\%$ oriented in the liquid crystal EBBA. (B) Spectrum A with protons decoupled. (Reprinted from Diehl \& Niederberger (1974), f. Magn. Reson., with permission from the copyright owner.)

so-called extreme-narrowing limit $\left(\omega^{2} \tau_{c}^{2} \ll \mathrm{I}\right)$ the deuteron relaxation times are given by (Abragam, I96I, p. 314)

$$
\frac{\mathrm{I}}{T_{1}}=\frac{\mathrm{I}}{T_{2}}=\frac{3}{8}\left(\frac{e^{2} q Q}{\hbar}\right)^{2} \tau_{c}
$$

The correlation time $\tau_{c}$ is a measure of the reorientation rate of the spherical molecule and is related to the microviscosity $\eta$ of the liquid according to

$$
\tau_{c}=\frac{4 \pi}{3} \frac{\eta r^{3}}{k T}
$$

$r$ is the radius of the sphere and $k T$ the thermal energy.

For the case of an asymmetric molecule the rates of rotation around the different molecular axes will not be identical. The rather complicated theory of anisotropic diffusion in pure liquids has been discussed by Huntress (1970). The practical aspects of the measurement and evaluation of such deuterium relaxation times have been reviewed by Mantsch et al. (1977).

Another complication is encountered in liquid crystalline mesophases where the deuteron is embedded in an ordered structure. Due to the unique molecular architecture of such systems the deuteron $\mathrm{C}-\mathrm{D}$ bond vector cannot tumble freely over all directions in space but executes 
angular oscillations of restricted amplitude. (Depending on the flexibility of the molecule the rates of reorientation around a given set of segment axes may or may not be anisotropic.) The usual linewidth expressions appropriate for pure liquids cannot be applied to such systems, since the relaxation rates are dependent on the ordering of the system. For sonicated single-walled vesicles Stockton et al. (1976) have derived the following linewidth expression

$$
\frac{\mathrm{I}}{T_{2}}=\frac{\mathrm{I}}{T_{1}}+\frac{9}{80}\left(e^{2} q Q / \hbar\right)^{2} S_{\mathrm{CD}}^{2} \tau_{v},
$$

where $S_{\mathrm{CD}}$ is the order parameter of the $\mathrm{C}-\mathrm{D}$ bond vector and $\tau_{v}$ is the correlation time for vesicle tumbling. The corresponding $T_{1}$-relaxation time is not dependent on the vesicle tumbling rate $\tau_{v}$ but on the rate of segment reorientation (J. Seelig, unpublished).

$$
\frac{\mathrm{I}}{T_{1}}=\frac{3}{8}\left(e^{2} q Q / \hbar\right)^{2} \tau_{c}\left(\mathrm{I}-S_{\mathrm{CD}}^{2}\right)
$$

The extreme-narrowing limit was assumed in deriving the latter two equations. Deuterium nmr spectra of vesicles exhibit only one resonance line since the vesicle rotation is fast enough to collapse the quadrupole splitting (Stockton et al. 1976). The order parameter $S_{\mathrm{CD}}$ in equations (7I) and (72) must therefore be derived from measurements of unsonicated samples.

Deuterium $T_{1}$ and $T_{2}$ relaxation time studies have also been performed for unsonicated lipid systems which do show quadrupole splittings. No simple expressions can be derived for $T_{1}$ and $T_{2}$ and the reader is referred to the literature (Egozy, Loewenstein \& Silver, I970; Jacobsen, Bildsoe \& Schaumburg, 1976; Jacobsen \& Schaumburg, 1976).

\section{ApPlication OF DEUTERIUM MAGNETIC RESONANCE} TO LIPID MEMBRANES

\section{A. Lipid bilayers composed of soap molecules}

Three lyotropic liquid crystals with lamellar structure have now been investigated in detail using deuterium nmr, namely (i) potassium lauratewater, (ii) sodium decanoate-decanol-water, and (iii) potassium palmitate-water. [In addition, deuterium $\mathrm{nmr}$ studies on lyotropic liquid crystals have been reported for hexagonal mesophases (Mely et al. 1975; 


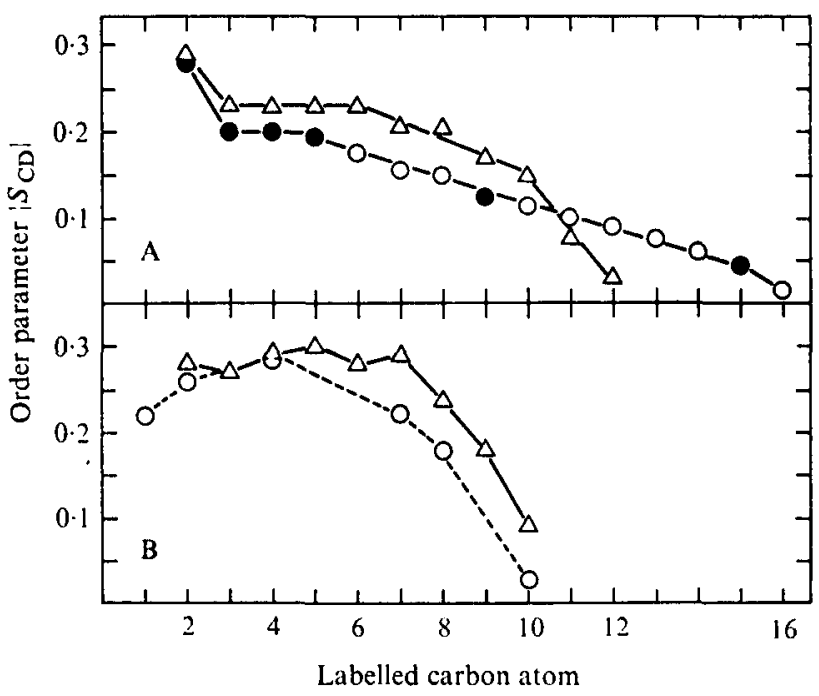

Fig. 15. Deuterium order parameters $S_{O D}$ for three bilayers composed of soap molecules as a function of the label position.

(A) Binary mixtures soap-water. $\triangle$, Perdeuterated potassium laurate with $21 \% \mathrm{H}_{2} \mathrm{O}$ at $82{ }^{\circ} \mathrm{C}$ (Charvolin et al. 1973 , fig. I); $\mathrm{O}$, perdeuterated potassium palmitate with $25 \% \mathrm{H}_{2} \mathrm{O}$ at $90{ }^{\circ} \mathrm{C}$ (Seelig \& Seelig, unpublished); - specifically deuterated potassium palmitate with $25 \% \mathrm{H}_{2} \mathrm{O}$ at $90{ }^{\circ} \mathrm{C}$ (Seelig \& Seelig, unpublished).

(B) Ternary mixture sodium decanoate (32 wt. \%), decanol $(38 \mathrm{wt} . \%$ ) and water $(30 \mathrm{wt} . \%) . \triangle$, Specifically deuterated sodium decanoate; $O$, specifically deuterated decanol. (Seelig \& Niederberger, 1974 $b$; Niederberger \& Seelig, 1974.)

Henriksson, Oedberg \& Eriksson, 1975), for a nematic-like mesophase (Long \& Goldstein, 1976) and for lamellar mesophases of as yet unknown X-ray structure (Reeves \& Tracey, 1975; Tracey \& Diehl, 1976).]

The binary mixture potassium laurate- $\mathrm{H}_{2} \mathrm{O}$ has been studied by Charvolin and co-workers with samples of perdeuterated lauric acid (Charvolin et al. 1973; Mely et al. 1975). In order to obtain well-resolved spectra the measurements were made with a planar oriented specimen. Seven quadrupole splittings were resolved under these conditions from which the order parameters pertaining to the different $\mathrm{CD}_{2}$ segments were evaluated. The assignment of the resonances was made by assuming that the order parameter decreases as the distance from the lipid-water interface increases. The variation of the order parameter with the position of the $\mathrm{CD}_{2}$ group along the alkyl chain is shown in Fig. I $5 \mathrm{~A}$. 
Figure ${ }_{5} \mathrm{~B}$ summarizes the results obtained for the ternary system sodium decanoate-decanol-water (Seelig \& Niederberger, 1974 $a, b$; Niederberger \& Seelig, 1974). A series of specifically deuterated decanoic acid and decanol molecules was synthesized for this purpose. Consequently the deuterium $\mathrm{nmr}$ measurements could be made with non-oriented samples and the assignment of the resonances was unambiguous. Fig. ${ }_{5} \mathrm{~B}$ reveals small, irregular variations of the order parameters so that for this particular system the assumption of a continuous decrease of the order parameter towards the bilayer interior is not valid. It may also be noted in Fig. ${ }_{5} \mathrm{~B}$ that the two types of molecules constituting the lipid bilayer are not entirely equivalent in their physical behaviour. Both chains have the same number of carbon atoms but decanol possesses one $\mathrm{CH}_{2}$ group more than decanoic acid so that the former penetrates deeper into the hydrophobic bilayer than the latter. If the results for decanoic acid are shifted by one carbon atom the two curves become almost superimposable on each other.

Apart from minor differences the qualitative behaviour of the two lipid phases is similar. Both bilayers exhibit essentially the same variation of the order parameter along the hydrocarbon chain. The order parameter remains fairly constant for most of the chain and only becomes much smaller for the terminal three or four segments.

These findings are at variance with previous spin label studies. For the ternary system sodium decanoate-decanol-water the spin label probes reveal a continuous decrease of the spin label order parameter (Seelig, I970; Seelig, Limacher \& Bader, 1972). The discrepancy between deuterium nmr and spin label epr must certainly be attributed to the perturbation of the bilayer by the bulky spin label group (see also p. 403). The perturbing influence of the oxazolidine ring is also demonstrated in monolayer studies which show that spin labelled fatty acids adopt two conformations at the air-water interface: erect, with only the carboxyl group in the air-water interface, and bent, with both the carboxyl group and the oxazolidine ring in this interface (Cadenhead \& Müller-Landau, I975).

The molecular interpretation of the deuterium order parameters can be accomplished at different levels of sophistication. Qualitatively the situation may be characterized as follows. The amphiphilic molecules are anchored with their polar groups in the lipid-water interface. The close packing of the polar heads imposes steric constraints on the hydrocarbon chains reducing the conformational freedom of the individual 


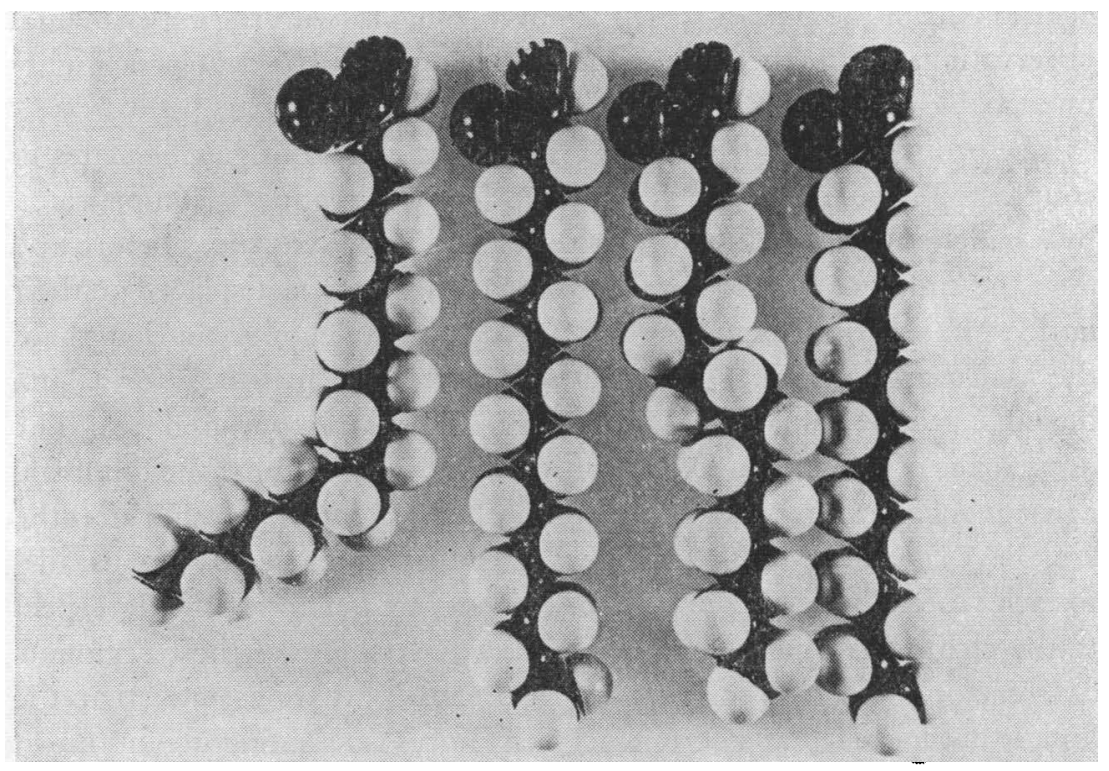

Fig. 16. Molecular models of various chain conformations. All trans conformation (2nd and $4^{\text {th }}$ chain), kink (3rd chain) and isolated gauche conformation (Ist chain).

chain molecules. This is the reason for the constant order parameter in the upper part of the chain. In the central part of the bilayer, which is the region of the free ends, the steric restrictions are relieved and the order parameter decreases.

The molecular origin of the chain flexibility is rapid isomerizations between trans and gauche conformations of the carbon-carbon bonds. For a preliminary discussion of the order parameters it suffices to consider just two types of conformational changes, namely (I) the formation of an isolated gauche conformation and (2) the formation of a kink $\left(g^{ \pm} \mathrm{tg}^{\mp}\right.$ sequence). Both structures are depicted in Fig. 16 . The formation of an isolated gauche state entails the collective reorientation of all segments between this gauche segment and the free methyl terminal of the chain (cf. Fig. r6). If the hydrocarbon chain is part of a lipid bilayer the formation of an isolated gauche state close to the polar interface is energetically unfavourable since it disrupts the parallel packing of the hydrocarbon chains. Also if the probability of occurrence of an isolated gauche state is assumed to be constant throughout the chain this would predict an exponential decrease of the order parameter towards the 
central part of the bilayer which is not in concord with the experimental observation. Thus the formation of isolated gauche states is highly improbable except in the central region of the bilayer.

The prominent feature of a kink is the occurrence of gauche states in coupled pairs so that a gauche rotation in one direction is compensated by a gauche rotation in the opposite sense. Trans-sequences before and after the kink thus remain parallel to each other but are displaced on their lattice site by approximately $I_{5} 5 \AA$. At the same time the length of the hydrocarbon chain is reduced by $1 \cdot 25 \AA$. The formation of a kink introduces only a small local defect into the bilayer (cf. Fig. I6). The occurrence of a kink at a fixed position in a trans chain has the following consequences for the order parameters. The segments before and after the kink have the same order parameters since their chain vectors are parallel to each other. At the kink itself two segments are bent by $60^{\circ}$ with respect to the chain axis thus reducing the order parameters of these segments by a factor $|S|=\left|\frac{1}{2}\left(3 \cos ^{2} 60^{\circ}-1\right)\right|=0.125$. Next we assume that the kink is not localized at a certain segment but is fluctuating up and down the chain. If the lifetime of the individual conformation is shorter than $\sim 10^{-6} \mathrm{sec}$ and if the formation of the kink occurs with equal probability regardless of the chain position, this model leads to a constant order parameter for all segments involved.

A kink is not the only conformational defect producing a constant order parameter. Sequences containing three or more trans segments between the coupled gauche rotations (so-called jogs, cf. Pechhold, 1968) have a similar effect, but their probability decreases rapidly with increasing size of the defect. Nevertheless jog-like structures make small but finite contributions to the total bilayer disorder.

For a quantitative insight into the microscopic structure of lipid bilayers the development of appropriate statistical theories is required. Several approaches have been published (Nagle, r 973 ; Belle \& Bothorel, 1974; Bothorel, Belle \& Lemaire, 1974; Scott, I974; Marčelja, 1974; McCammon \& Deutch, 1975; Jacobs, Hudson \& Andersen, 1975) which discuss in detail the melting behaviour of lipid-water systems. However, an explicit treatment of order parameters is only included in the theories of Marčelja and of Bothorel. Marčelja's molecular field calculation explains remarkably well the deuterium order parameters of the sodium decanoate-decanol bilayer using just two adjustable parameters. Details of this approach will be given in the next section in connection with phospholipid bilayers. 
Before concluding this section on soap-like bilayers we shall briefly examine the binary system potassium palmitate-water which has been studied using perdeuterated as well as selectively deuterated palmitic acid (Seelig \& Seelig, unpublished results; Davis \& Jeffrey, 1977). At $90^{\circ} \mathrm{C}$ this bilayer exhibits a monotonous decrease of the deuterium order parameter when the label is moved towards the methyl terminal (Fig. I 5 A). The plateau region characteristic of the other two bilayers has almost completely disappeared. Phenomenologically this behaviour may be explained by the rather large effective surface area of potassium palmitate at $90^{\circ} \mathrm{C}$. It has been pointed out by Mely et al. (1975) that an increase of the surface area per lipid molecule implies a less ordered bilayer since the expansion of the lattice leads to larger conformational freedom for the hydrocarbon chains. From the work of Gallot \& Skoulious (I966) the surface area of the potassium palmitate system can be estimated to be $38 \AA^{2}\left(25 \% \mathrm{H}_{2} \mathrm{O}, 90^{\circ} \mathrm{C}\right)$ compared to $34 \AA^{2}$ for the potassium laurate system and only $25 \AA^{2}$ for the sodium decanoate-decanol bilayer. On the other hand, if the surface area of the potassium laurate bilayer is enlarged to $39 \AA^{2}$ (using a higher temperature and an increase in water content) the same variation of the deuterium order parameter with the chain position as in the potassium palmitate system is observed (cf. Mely et al. 1975, fig. 3).

\section{B. Phospholipid bilayers}

The physical-chemical properties of phospholipid bilayers have recently been reviewed by Lee (1975). Special consideration was given to the application of spin label epr and carbon- $\mathrm{I} 3 \mathrm{nmr}$ techniques and this article may be consulted for principal references to both methods which have strongly influenced the current picture of the molecular organization of phospholipid bilayers. The validity of the spin label results must however be questioned since the label group can 'significantly perturb a normal lipid environment. Consequently, some quantitative observations that used spin label techniques need revision while others may be reduced to the level of qualitative prediction' (Cadenhead \& MüllerLandau, 1975). The problems associated with the use of spin labels have already been recognized by Lee (1975) who points out three areas where modifications of the spin label picture might be required, namely (i) the tilt angle of the hydrocarbon chains ('bent' fatty acid chains), (ii) the ordering profile ('flexibility gradient'), and (iii) the polarity profile (water penetration into the bilayer). We shall discuss the first two of these 


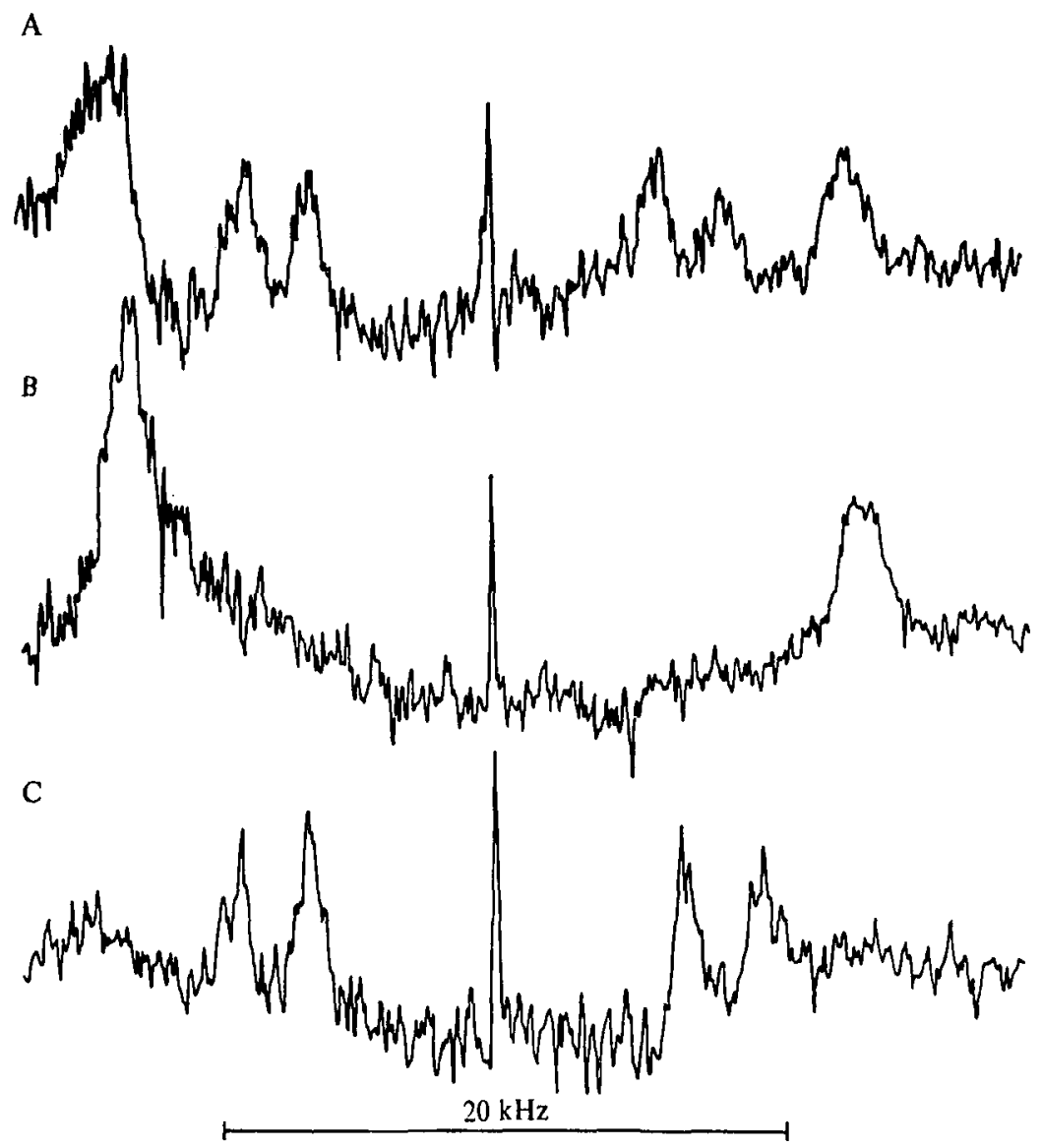

Fig. 17. Deuterium magnetic resonance spectra of bilayers of dipalmitoyl-3$s n$-phosphatidylcholine measured with a superconducting magnet $(4 \mathrm{I} \cdot 4 \mathrm{MHz})$ at $50^{\circ} \mathrm{C}$. (A) $I, 2-\left[2^{\prime}-d_{2}\right]$-dipalmitoyl-3-sn-phosphatidylcholine. (B) $I\left[z^{\prime}-d_{2}\right]$ palmitoyl-2-palmitoyl-3-sn-phosphatidylcholine. (C) I-palmitoyl-2 $\left[2^{\prime}-d_{2}\right]$ palmitoyl-3-sn-phosphatidylcholine. (Seelig \& Seelig (I975), Biochim. biophys. Acta; reprinted with permission of the copyright owner.)

problems as we progress in the description of the application of deuterium $\mathrm{nmr}$ to phospholipid bilayers.

\section{Hydrocarbon region}

Fig. I7 shows a comparison of three deuterium resonance spectra of dipalmitoyl-3-sn-phosphatidylcholine (DPPC) with the deuterium labels at the $\mathrm{C}-2$ segment of the palmitic acyl chains. Three separate signals are observed, the largest $(\sim 30 \mathrm{kHz})$ being due to the chain attached at 
carbon atom I of the glycerol (chain I), the two smaller ones (23 and ${ }_{14} \mathrm{kHz}$ ) arising from the other chain (chain 2) (Seelig \& Seelig, I975).

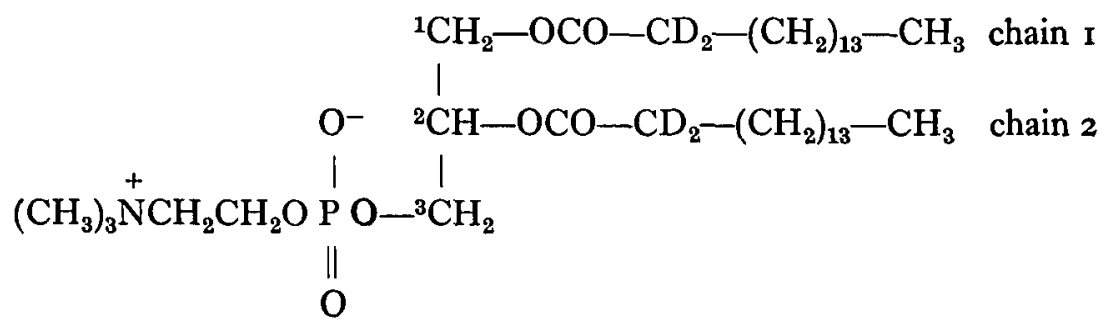

This result is indicative of different orientations for the beginnings of the two chains. In most parts of the bilayer both fatty acyl chains are extended perpendicular to the bilayer surface except for the first segment of chain 2 which is orientated essentially parallel to the bilayer surface (Seelig \& Seelig, 1975; Schindler \& Seelig, 1975). This view is supported by X-ray diffraction studies of crystalline bilayers of related dilauroylphosphatidylethanolamine which show that chain 2 is bent at the first segment and penetrates less deeply into the bilayer than chain I (Hitchcock et al. 1974). The conformational twisting in the vicinity of the polar group is also sensed in other parts of the hydrocarbon chains, since two quadrupole splittings are observed for the $\mathrm{C}-3$ segment and then again for the $\mathrm{C}-\mathrm{IO}$ to C-I 5 segments (Seelig \& Seelig, I974 $b$, table I). Even though the difference between the two splittings is rather small $(2-3 \mathrm{kHz})$ the data clearly demonstrate that the two fatty acyl chains of a phospholipid molecule are not entirely equivalent. Corresponding segments of the two chains are positioned at slightly different distances from the lipid-water interface and experience different fluctuating movements. This effect is temperature dependent and the difference between the two chains increases as the system approaches the gel-to-liquid crystal transition point (Seelig \& Seelig, 1974 $b$, table I).

For the quantitative analysis of the deuterium quadrupole splittings the order parameters $S_{\mathrm{CD}}$ or $S_{\text {mol }}$ may be used, these being the order parameters of the C-D bond and of the whole segment, respectively. The segment direction is defined here by the normal to the plane spanned by the two CD bonds of a methylene segment. With this definition the orientation of the individual chain segments will coincide with the long molecular axis if the chain is frozen in the extended all-trans conformation. Assuming an axially symmetric movement of the $\mathrm{CD}_{2}$ segment 
around the long molecular axis ( $\rho$-axis of fig. 13) the two order parameters are related to each other according to (cf. equation (68); $\beta^{*}=90^{\circ}$,

$$
S_{\mathrm{mol}}=-2 S_{\mathrm{CD}}^{-}
$$

Since the average orientation of a $\mathrm{C}-\mathrm{D}$ bond is perpendicular to the bilayer normal (cf. below) it is safe to assume that $S_{\mathrm{CD}}$ is negative, so that $S_{\mathrm{mol}}$ becomes positive. The advantage of using $S_{\mathrm{mol}}$ is that it provides a measure for the ordering of the chain axis and that it is also directly comparable to the order parameter determined with spin labelled fatty acids.

The results of the two methods for bilayers of DPPC are shown in Fig. 18. With the exception of the terminal 6 or 7 segments only a slight variation of the deuterium order parameter along the chain is observed, in contrast to the large variation measured using spin probes. A qualitatively similar result has been obtained for bilayers of dimyristoyl-3sn-phosphatidylcholine, whose deuterium resonance spectra were recorded for the lipid with perdeuterated fatty acyl chains (Oldfield et al. I971).

Before discussing the deuterium results any further a brief remark about order and fluidity is necessary. Fluidity in the sense used here refers to the rate of motion but not to the ordering of the molecular system. No simple relationship between the two quantities is known for a lipid bilayer. The independence of the two parameters is illustrated by comparing the phospholipid bilayer (Fig. I8) with the sodium decanoate-decanol bilayer (Fig. ${ }_{5} \mathrm{~B}$ ). In the latter system the average order parameter in the constant region is $S_{\mathrm{mol}} \approx 0.6$ while the microviscosity is found to be about 0.05 poise (Schindler \& Seelig, 1973, 1974). In the DPPC bilayer the microviscosity is probably more than ten times larger as judged from epr diffusion experiments (Träuble \& Sackmann, 1972; Devaux \& McConnell, 1972) but the system is at the same time less ordered $\left(S_{\mathrm{mol}} \approx\right.$ $0.4-0.5)$. Hence the soap-like bilayer is well ordered and highly fluid while the phospholipid bilayer is less ordered and less fluid. This demonstrates that the fluidity of a bilayer to a certain extent is independent of the order parameter and must be determined by a separate measurement. The necessary information could be provided by proton, carbon-I 3 or deuterium relaxation times (Horwitz et al. 1973; Horwitz, Horsley \& Klein, I972; Metcalfe et al. I971 ; Metcalfe, Birdsall \& Lee, 1973; Chan, Seiter \& Feigenson, 1972; Chan et al. 1973; Stockton et al. 1976; Boden et al. 1976). The interpretation of $T_{1}$ and $T_{2}$ relaxation times is however difficult since in an oriented system $T_{1}$ and $T_{2}$ depend upon the order 


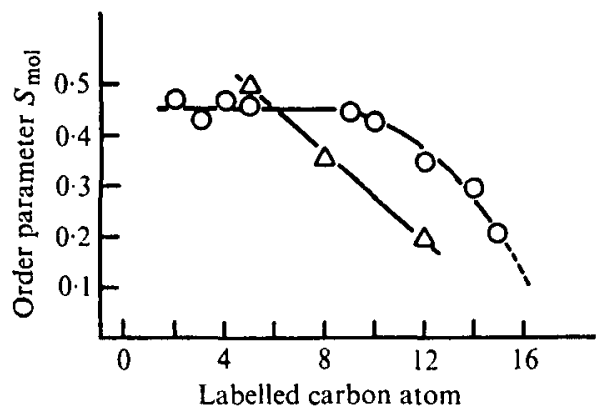

Fig. 18. Order parameter $S_{\text {mol }}$ of bilayers of dipalmitoyl-3-sn-phosphatidylcholine as a function of the position of the labelled carbon atom at $4 \mathbf{I}{ }^{\circ} \mathrm{C}$. $O$, Deuterium data (Seelig \& Seelig, 1974b); $\triangle$, spin label data (Hubbell \& McConnell, 1971).

as well as on the rate of motion. Nevertheless the available data seem to suggest a fairly constant rate of segment reorientation for the region of the chain up to $n=10$ and an increase of the motional rate beyond this point. Since the change in fluidity is confined to a narrow region in the centre of the bilayer it may be questioned if the term 'fluidity gradient' is especially well suited to characterize this bilayer property.

We proceed now to a discussion of the hydrocarbon chain ordering. $\mathrm{X}$-ray diagrams of lipid bilayers in the liquid crystalline state (so-called $L_{\alpha}$-phase; Luzzati, I968) are characterized by a broad band at $(4 \cdot 6 \AA)^{-1}$ which is correlated with the chain-chain separation. The marked similarity of this band to that from liquid paraffins leads to the conclusion that 'the $\alpha$-conformation can be visualized as highly disordered, like that of a liquid paraffin, still with the average of the chain orientations perpendicular to the lipid water interface' (Tardieu, Luzzati \& Reman, 1973). The concept of disordered chains oriented perpendicular to the bilayer surface is fully supported by deuterium nmr. Moreover this method leads to ideas about the chain configuration and packing which are not obtainable by other techniques.

Firstly, studies with orientated multilayers of $\mathrm{C}-5$ deuterated DPPC show that the quadrupole splitting collapses if the bilayers are inclined at the magic angle with respect to the magnetic field (Seelig \& Seelig, $1974 a$ ). It can be concluded that the axis of motional averaging is identical with the bilayer normal, i.e. the rotation of the lipid molecule as a whole and, in part, the motions about $\mathrm{C}-\mathrm{C}$ bonds within the fatty acyl chains are axially symmetric around the bilayer normal. This result is important because it excludes any collective tilt of the hydrocarbon chains with a 
lifetime of longer than about ${ }^{10^{-6}}$ sec. A similar result was obtained for soap-like bilayers (Charvolin et al. 1973; Seelig \& Niederberger, 1974a), which implies that in all these systems 'it would be misleading to talk of any long-lived net bend in the chains' (Lee, 1975).

A collective tilt of the hydrocarbon chains has been inferred from spin label studies of egg-yolk lecithin (McFarland \& McConnell, 1971). This lipid contains unsaturated fatty acids of different chains length and may exhibit a behaviour different from the synthetic lipids discussed so far. On the other hand it cannot be excluded that the cooperative tilt is an artifact introduced by the spin label group. In this respect it is interesting to note that a tilt is measured only with spin-labelled phospholipids but not with spin-labelled fatty acids. However, even if the spin label result is correct for egg-yolk lecithin, deuterium nmr at least demonstrates that a cooperative tilt is not a general membrane phenomenon.

The second information contained in the deuterium quadrupole splittings concerns the configuration of the fatty acyl chains. Only the average chain configuration is detected since the jump frequency at which the molecule jumps from one configuration to the other $\left(c \cdot{ }^{10^{10}} \mathrm{sec}^{-1}\right)$ is fast compared to the deuterium resonance frequency $\left(c .10^{7} \mathrm{sec}^{-1}\right)$. In this respect the method is related to $\mathrm{X}$-ray diffraction where the broad band at $(4.6 \AA)^{-1}$ represents only the average value of the interchain separation. However, while $\mathrm{X}$-ray diffraction measures an average over the whole bilayer cross-section, deuterium order parameters pertain to averages over the movements of individual segments, thus yielding a picture of the chain configuration at a much higher resolution.

Fig. 18 shows that the order parameters for DPPC at temperatures just above the phase transition are about 0.5 or less indicating a considerable chain flexibility. If the all-trans conformation (with $S_{\mathrm{mol}}=\mathrm{I}$ ) is referred to as the ordered state, then the fatty acyl chains in a bilayer may be called disordered, since they contain an appreciable number of gauche conformations. This is also supported by Raman measurements which have provided direct evidence for the existence of gauche conformations in lipid bilayers (Lippert \& Peticolas, 1971; Mendelsohn, 1972; Gaber \& Peticolas, 1977). The notion of disordered fatty acyl chains is further substantiated by a quantitative comparison of trans and gauche probabilities in liquid paraffin chains and in lipid bilayers. The configuration of a hydrocarbon chain in solution is determined essentially by intramolecular forces, i.e. the bond rotation potential for trans-gauche transitions and steric interactions between neighbouring chain segments 
(cf. Flory, rg69). The segment interactions are cooperative and are normally taken into account by applying a linear Ising model. On the basis of this model, which is now well established in polymer chemistry, the probabilities of trans and gauche conformations in a liquid polymethylene chain are found to be $p_{t} \approx 0.6$ and $p_{g} \approx 0.4$, respectively (Flory, 1969, p. 82). These numbers refer to temperatures of $400-450^{\circ} \mathrm{K}$; at room temperature the trans-state is slightly more populated $\left(p_{t} \approx 0.65\right)$. In a bilayer the chain configuration is determined by intramolecular as well as intermolecular forces. According to Marčelja (1974) the total energy, $E^{(1)}$, of a hydrocarbon chain with the configuration (i) may be written as the sum of three contributions

$$
E^{(\mathrm{i})}=E_{\mathrm{int}}^{(\mathrm{i})}+E_{\mathrm{disp}}^{(\mathrm{l})}+\gamma A^{(\mathrm{i})} .
$$

The first term, $E_{\mathrm{int}}^{(1)}$, represents the intramolecular energy of the free chain. The second contribution, $E_{\text {dlsp }}^{(i)}$, summarizes the dispersive interactions, i.e. the van-der-Waals attractions between the chains. These interactions are characterized by the coupling constant $V_{0}$, which is an adjustable parameter. The third term, $\gamma A^{(1)}$, accounts for various types of interactions such as steric chain-chain repulsions and electrostatic interactions among the head groups. $A^{(\mathrm{i})}$ is the effective cross-sectional area of the hydrocarbon chain with the configuration (i), while $\gamma$ may be called lateral pressure or surface energy. $\gamma$ is the second free parameter in the theory. The partition function of the system and average chain properties can then be calculated by means of a molecular field approximation. This model has been used to analyse the deuterium order parameters in DPPC bilayers (Schindler \& Seelig, 1975). The experimental values could be fit for $V_{0}=590 \mathrm{cal} / \mathrm{mole}$ and $\gamma=18.5 \mathrm{erg} / \mathrm{cm}^{2}$ although these are not the only values possible. With these parameters the model predicts a transition temperature of $39.5^{\circ} \mathrm{C}$ for the gel-to-liquid crystal phase transition, an entropy change for the transition of $\mathrm{I} \cdot \mathrm{r}_{5}$ e.u. per $\mathrm{CH}_{2}$ group and an average cross-sectional area per lipid molecule of $58.6 \AA^{2}$, in remarkable agreement with calorimetric and $X$-ray diffraction measurements. Using the same set of parameters the probability of a carboncarbon bond"to occur in the trans (gauche) conformation is then found to be $p_{t}=0.69\left(p_{g}=0.3 \mathrm{I}\right)$ at $4 \mathrm{I}{ }^{\circ} \mathrm{C}$, which is not significantly different from the corresponding probabilities for the liquid paraffin chain. Therefore, on the average, 9.5 bonds of a palmitic acyl chain are in the trans state while 4.5 are in the gauche state, regardless of whether the molecule is incorporated into a bilayer or is dissolved in a paraffinic liquid. Due 


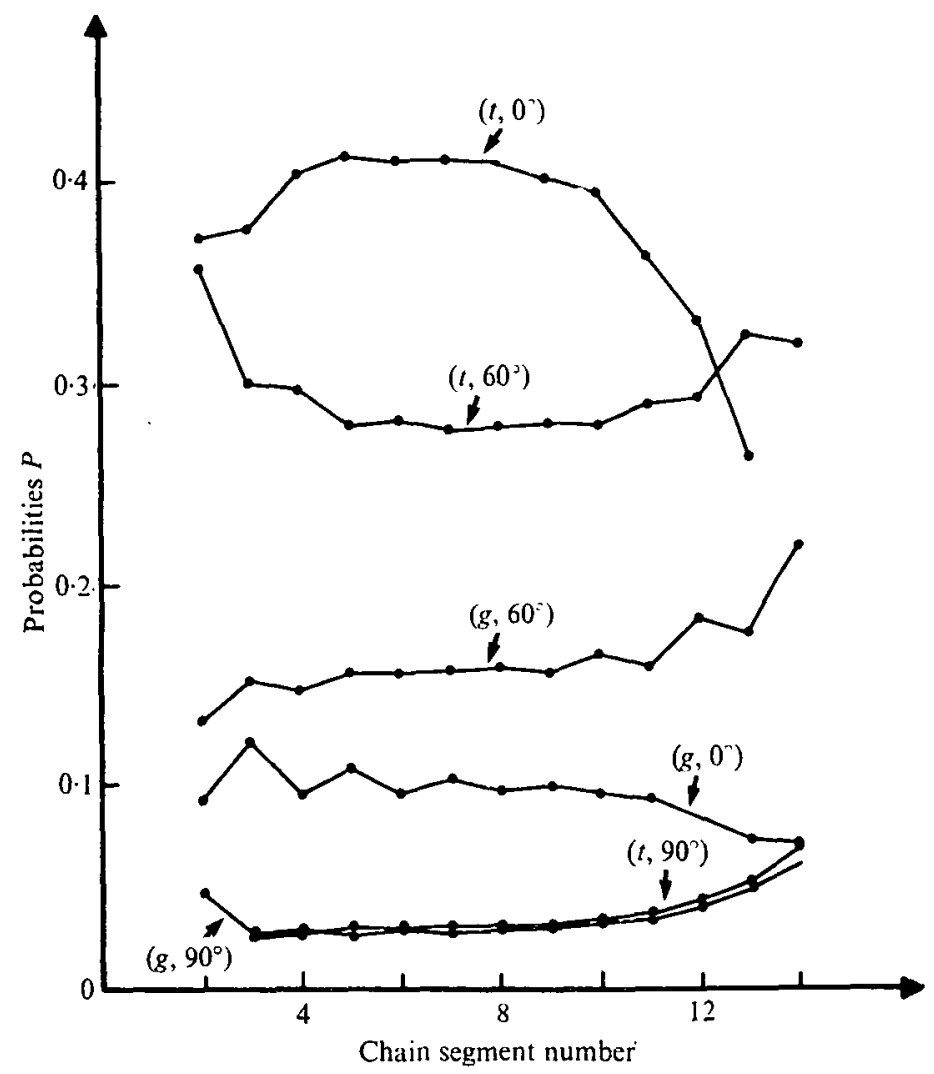

Fig. 19. Probabilities of segment orientations as a function of the segment position in the bilayer (Schindler \& Seelig (1975), Biochemistry; reprinted with permission of the copyright owner).

to this similarity in the average number of gauche conformations it seems justified to characterize the chain configuration in a phospholipid bilayer as disordered.

This does not imply, however, that the chain configurations in the two phases are completely identical. In the liquid phase both ends of the chain are free to move and the statistical averages do not discriminate in favour of one end of the chain over the other. For example, a calculation of the probability of trans or gauche states as a function of segment position yields almost identical probabilities for all segments (Flory, I969, p. 82). In the bilayer, however, one end of the chain is anchored in the lipid-water interface, endowing the chain with a distinct polarity. Consequently, the probability of occurrence of a trans or gauche state becomes 


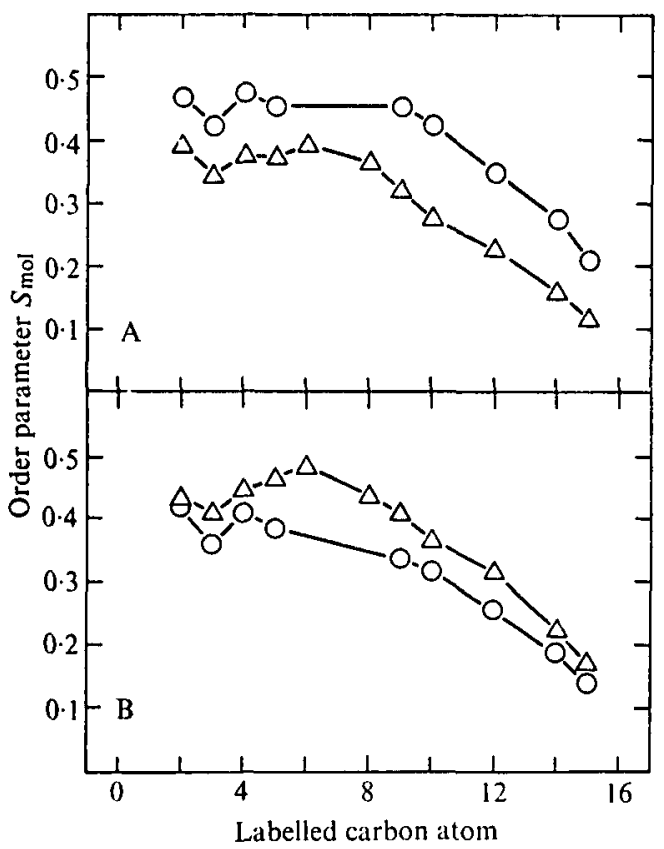

Fig. 20. Influence of cis-double bonds on the ordering of the hydrocarbon chains. Comparison of the deuterium order parameter $S_{\mathrm{mol}}$ for bilayers of dipalmitoyl-3-sn-phosphatidylcholine $(O)$ and I-palmitoyl-2-oleoyl-3-snphosphatidylcholine $(\triangle)$ (phase composition $51.5 \mathrm{wt}$ \% water $/ 48.5 \mathrm{wt}$. $\%$ lipid). (A) Both bilayers at identical temperatures of $42^{\circ} \mathrm{C}$. (B) Both bilayers at equal temperatures relative to the respective phase transition temperatures. $\triangle$, POPC at $14{ }^{\circ} \mathrm{C}=T_{c}+19^{\circ} \mathrm{C} ; O, D P P C$ at $60^{\circ} \mathrm{C}=T_{c}+19^{\circ} \mathrm{C}$. (Seelig $\&$ Seelig (1977), Biochemistry; reprinted with permission of the copyright owner.)

dependent on the position of the segment within the bilayer. Furthermore, it turns out that trans and gauche segments must be differentiated according to their orientation with respect to the bilayer normal, since different orientations have different energies. If it is assumed that the first segment of the hydrocarbon chain is parallel to the bilayer normal, then only a few segment orientations are possible on the basis of transgauche isomerizations: the segments are inclined to the bilayer normal at angles of either 0,60 or $90^{\circ}$ (cf. Seelig \& Seelig, 1974 $b$; Schindler \& Seelig, 1975). Since the conformational state in each of these orientations may be either trans or gauche, this leads to altogether six types of segments. Their probabilities as a function of the segment position are plotted in Fig. I9. Inspection of Fig. I9 reveals that the largest probability 
must be assigned to trans segments parallel to the bilayer normal. This indicates that the parallel alignment of the hydrocarbon chains is the energetically most favourable situation. Fig. I9 also shows that the total probability of the trans conformations $\left(p_{t, 0^{\circ}}+p_{t, 60^{\circ}}+p_{t, 80^{\circ}}\right)$ remains almost constant throughout the bilayer. The loss of order in the central part of the bilayer is essentially due to a decrease in the number of $\left(t, 0^{\circ}\right)$ elements and a simultaneous increase in the number of $\left(t, 60^{\circ}\right)$ and $\left(g, 60^{\circ}\right)$ elements.

It is equally simple to calculate the probabilities of kinks $\left(g^{+} t g^{-}\right.$, $\left.g^{-} t g^{+}\right)$or jogs $\left(2 g 2 \mathrm{jog}=g^{+} t t t g^{-}\right)$. As has already been pointed out, these sequences are of importance since they pack very well into the bilayer and leave the chains essentially parallel to each other. The numerical computations yield approximately 0.6 kink and 0.2 jog per chain. The kink and the jog are found to be the most important single contributions to the chain flexibility which together account for approximately $50 \%$ disorder. The remaining disorder comes from small but nevertheless finite contributions from a large number of other conformational defects. Thus even in the region of constant order parameter it is not sufficient to postulate kinks and $2 g 2$ jogs, but other configurations must also be included. The same calculation also indicates that the probability of a kink, $p_{\mathrm{k} \text { In }}$, depends on the location of the kink in the bilayer and that it decreases in the central region of the bilayer.

Phospholipids isolated from biological membranes contain a rather large percentage of unsaturated fatty acids. A deuterium magnetic resonance study pertaining to the influence of cis-double bonds on the chain ordering has been performed with r-palmitoyl-2-oleoyl-3-snphosphatidylcholine (POPC) (Seelig \& Seelig, 1977). In Fig. 20 the deuterium order parameter $S_{\mathrm{mol}}$ of this system is compared to that of bilayers prepared from dipalmitoyl-3-sn-phosphatidylcholine (DPPC). In both lipids the deuterium label is attached to the saturated chain (chain I; cf. p. 395). At equal temperatures the unsaturated bilayer is found to be less ordered than the saturated phase (Fig. $20 \mathrm{~A}$ ). This is a direct consequence of the difference in the gel-to-liquid crystal transition temperatures which for POPC and DPPC are -5 and $+4 \mathrm{I}^{\circ} \mathrm{C}$, respectively. From a physical point of view it is more realistic to compare order parameters at the same relative temperature with respect to the transition point (Fig. 20 B). Under this condition both bilayers have almost identical segmental order parameters from $\mathrm{C}-2$ to $\mathrm{C}-4$ and again from $\mathrm{C}-9$ to $\mathrm{C}-15$. In the intermediate region the unsaturated bilayer is however clearly 
more ordered than the saturated. This suggests a local rigidity caused by the cis-double bond which reduces the motional freedom of the $\mathrm{CH}_{2}$ segments of neighbouring chains.

Natural lipid mixtures and biological membranes are not generally amenable to direct deuterium substitution. As an alternative, deuterated fatty acids can be intercalated physically into phospholipid bilayers. This approach was chosen to study bilayers of egg-yolk lecithin (Stockton et al. 1976). The shapes of the curves drawn through the deuterium order parameters resemble those observed for dipalmitoyl-3-sn-phosphatidylcholine and I-palmitoyl-2-oleoyl-3-sn-phosphatidylcholine, though finer details of the structure are smoothed out. The reason for this is probably the rather high percentage of fatty acids incorporated, which is necessary to obtain reasonable spectra. [The transition temperature of dipalmitoylphosphatidylcholine, as observed by differential scanning calorimetry, is raised from $4 \mathrm{I}^{\circ} 4$ to $61.5^{\circ} \mathrm{C}$ by addition of palmitic acid at a mole fraction of 0.67 (Mabrey \& Sturtevant, 1977).] By comparing the linewidth and relaxation times of sonicated and non-sonicated bilayers it could also be demonstrated that the molecular motion and order in singlewalled bilayer vesicles are very similar to those in lamellar multilayers (Stockton et al. r976).

Let us briefly return to spin labels. Previous spin label studies of phospholipid bilayers have led to a different model for the chain ordering. It was found that in bilayers with saturated hydrocarbon chains (DPPC-bilayers; Hubbel\& McConnell, 1971) as well as in bilayers prepared from natural lipids (eggyolk lecithin; Jost et al. 197I; McFarland \& McConnell, 1972) the spin label order parameter decreases rapidly as the paramagnetic nitroxide group approaches the centre of the bilayer. This behaviour which parallels that of spin probes incorporated into soap bilayers has been referred to as 'flexibility gradient'. The deuterium resonance experiments with dipalmitoyl-3-snphosphatidylcholine and I-palmitoyl-2-oleoyl-3-sn-phosphatidylcholine show, however, that the deuterium order parameters are constant in about two thirds of the bilayer and in view of these findings the 'flexibility gradient' is probably a poor term to characterize the bilayer.

It has also been argued that the perturbation of the bilayer by the spin label group is not serious and that the main source of discrepancy is the different time scale of the two methods (Gaffney \& McConnell, 1974; McConnell, 1976). Spin label epr detects fast motions and the spin label order parameter comprises fluctuations with frequencies $\geqslant 10^{8} \mathrm{~Hz}$. Deuterium nmr has a lower time resolution and the deuterium order parameter refers to fluctuations with frequencies $\geqslant 10^{5} \mathrm{~Hz}$. Motions with frequencies of $10^{5}-10^{7} \mathrm{~Hz}$ are therefore not included in the spin label order parameter but are included in the deuterium 
order parameter. In fact, if the average angular fluctuation in the $10^{5}-10^{7} \mathrm{~Hz}$ frequency range is given by $\overline{\cos ^{2} \alpha}$, then the deuterium order parameter $S_{\text {mol }}$ is related to the spin label order parameter $S_{3}$ according to

$$
S_{\mathrm{mol}} \approx(\mathrm{r} / 2)\left(3 \overline{\cos ^{2} \alpha}-\mathrm{I}\right) S_{3} \text {. }
$$

On the basis of this time scale effect the deuterium order parameter must therefore be expected to be smaller than the corresponding spin label order parameter. However, a detailed comparison of the two methods for two identical bilayer systems leads to just the opposite result. In both the soaplike bilayer of sodium decanoate-decanol (Seelig \& Niederberger, $1974 b$, fig. 2 ) and in the phospholipid bilayer of DPPC (fig. I 8) the deuterium order parameters are found to be larger than the spin label order parameters. This rules out the time scale argument at least for these two bilayers.

To summarize the above information about the hydrocarbon region in the liquid-crystalline state, the weight of evidence seems to favour liquid-like, disordered hydrocarbon chains containing, on the average, almost the same number of gauche conformations as the corresponding liquid paraffins. All parts of the hydrocarbon chains undergo rapid rotational transitions. In about two thirds of the bilayer cross-section coupled gauche rotations (kinks, jogs) are preferred in order to facilitate a mostly parallel packing of the chains, leading to the picture of disordered chains in an ordered bilayer. In the central part of the bilayer the steric constraints are relieved and the probability of segment orientations other than parallel increases distinctly. However, since all segments execute rapid angular oscillations it would be misleading to describe this change in the order parameter as a gradual crystalline-to-fluid transition within the bilayer. The two hydrocarbon chains of a phospholipid molecule are not completely equivalent, since the beginnings of the two chains have different orientations with respect to the bilayer surface. For the bilayers investigated so far, the deuterium magnetic resonance data seem to disprove the existence of any significant long-lived or short-lived cooperative tilt of the hydrocarbon chains in the liquid crystalline phase.

Below the gel-to-liquid crystal transition point the hydrocarbon chains are frozen into a rather rigid all-trans conformation and a variety of different structures have been observed with $\mathrm{X}$-ray diffraction (cf. Tardieu et al. 1973). A unique feature of synthetic lecithins with two identical, saturated hydrocarbon chains like dimyristoyl-3-sn-phosphatidylcholine or dipalmitoyl-3-sn-phosphatidylcholine is the occurrence of a small endothermic pretransition a few degrees below the main transition. For dipalmitoyl-3-sn-phosphatidylcholine it has recently been 
suggested on the basis of $\mathrm{X}$-ray evidence that below this pretransition at $35^{\circ} \mathrm{C}$ 'the chains are fully extended, packed in a hexagonal lattice but tilted with respect to the plane of the bilayer. Between 35 and $42{ }^{\circ} \mathrm{C}$ the chains are similarly packed but oriented perpendicular to the bilayer plane. Above $42{ }^{\circ} \mathrm{C}$ the chains are melted or disordered' (Rand, Chapman \& Larsson, 1975). A different result has been found by Janiak, Small \& Shipley (1976). According to these authors 'the pretransition is associated with a structural transformation from a one-dimensional to a twodimensional monoclinic lattice consisting of lipid lamellae distorted by a periodic ripple. The hydrocarbon chains remain tilted in the temperature range intermediate between the pretransition and chain melting transition.' No well-resolved deuterium resonance spectra have as yet been obtained for phospholipid bilayers in the gel-state, since the lines become too broad. From the broad spectra found for perdeuterated dimyristoyllecithin it could be concluded, however, that some motion is occurring even in the gel state (Oldfield et al. 1971). This has been confirmed by a more detailed study on a potassium stearate bilayer in the gel state (Mely \& Charvolin, 1977).

The influence of cholesterol on the hydrocarbon chain ordering has also been investigated using deuterium magnetic resonance. Perdeuterated phospholipids (Oldfield et al. 197I) and perdeuterated fatty acids (Stockton \& Smith, 1976) as well as selectively deuterated phospholipids and cholesterol Gally et al. 1976) have been employed. Due to the presence of cholesterol the quadrupole splittings of fatty acyl chain segments adjacent to the rigid sterol frame increase to about twice the value found in the cholesterol-free bilayer. The order parameter derived from deuterated cholesterol is identical to that of the neighbouring paraffinic chains.

\section{Structure and flexibility of the polar head groups}

Our understanding of the structure of polar head groups is generally rather limited (cf. Lee, I975). Recent progress has been made, however, in the study of the ethanolamine and choline head group. In the following discussion we shall confine ourselves to lipid bilayers carrying either one of these polar groups.

A precise picture of the molecular conformation of the ethanolamine head group has been derived for single crystals of dilauroyl-DL-phosphatidylethanolamine (Hitchcock et al. 1974) and L- $\alpha$-glycerylphosphorylethanolamine (DeTitta \& Craven, 1971, 1973) using X-ray diffraction 


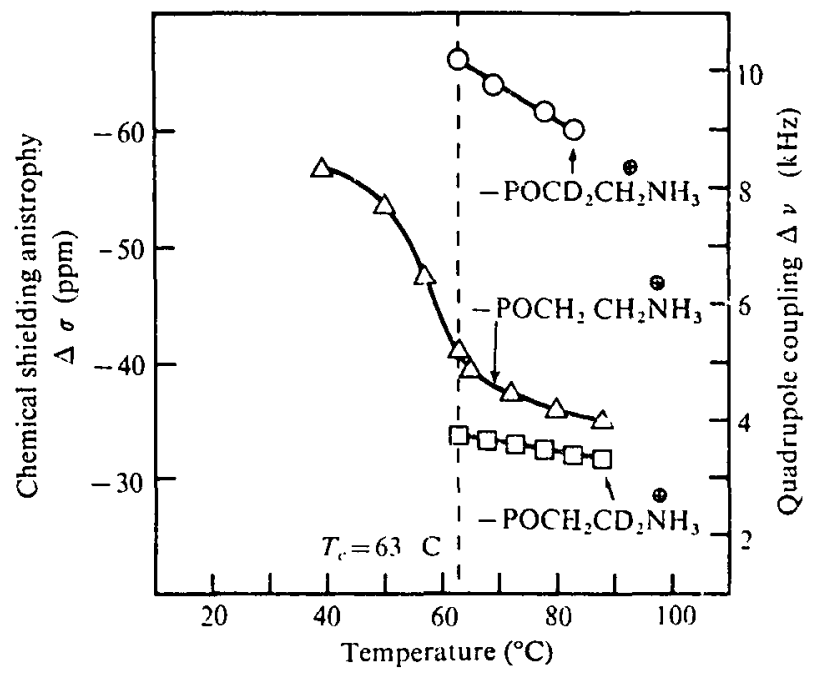

Fig. 2r. Variation of the quadrupole splittings and the phosphorus shielding anisotropy of multilayers of dipalmitoyl-3-sn-phosphatidylethanolamine with temperature. (Seelig \& Gally (1976), Biochemistry; reprinted with permission of the copyright owner.)

techniques. Common to both structures is the gauche conformation of the $\mathrm{O}-\mathrm{C}-\mathrm{C}-\mathrm{N}^{+}$

$\mathrm{O}^{13}$

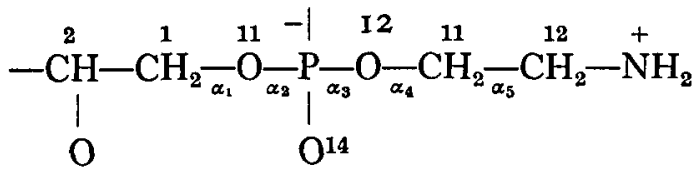

system (torsion angle $\alpha_{5}$ ) and the gauche-gauche rotation of the phosphodiester linkage $\left(\alpha_{2}, \alpha_{3}\right)$ leading to a 'boomerang' shape of the ethanolamine group in the crystal (DeTitta \& Craven, I97I). The nomenclature used here is that introduced by Sundaralingam (1972). The torsion angles $\alpha_{i}$ are measured from the cis-planar configuration.

It has been suggested that the crystal conformation most likely will also be exhibited in the liquid crystalline state characteristic of biological membranes. This prediction can be tested using deuterium magnetic resonance. For this purpose dipalmitoyl-3-sn-phosphatidylethanolamine was selectively deuterated at the two ethanolamine carbon atoms and the residual deuterium quadrupole splittings measured as a function of temperature. In addition the motion of the phosphate group was followed using phosphorus-3 I magnetic resonance, where the structural information is contained in the chemical shielding anisotropy (Niederberger 
\& Seelig, 1976). The variation of the deuterium quadrupole splittings, $\Delta \nu$, and the phosphorus chemical shielding anisotropy, $\Delta \sigma$, are plotted in Fig. 21 as a function of temperature (Seelig \& Gally, 1976). The following conclusions can be inferred from these experiments. Firstly, the motions of the phosphate group and of the two carbon atoms of the ethanolamine moiety are clearly anisotropic. Secondly, the freezing of the hydrocarbon chains is reflected in the freedom of rotation of the whole polar group, since the chemical shielding anisotropy, $\Delta \sigma$, decreases by about $20 \mathrm{ppm}$ and since the deuterium signals are completely lost if the temperature is lowered beyond $63{ }^{\circ} \mathrm{C}$. This transition occurs exactly at the gel-to-liquid crystal transition point as determined by differential scanning calorimetry (Vaughan \& Keough, 1974). Below this critical temperature the ethanolamine group is presumably locked into a rigid conformation which together with a reduction in the reorientation rate makes the quadrupole splittings too large and the lines too broad to be detected. Thirdly, it follows from studies with planar oriented multilayers that the motion of the whole polar group is axially symmetric around an axis perpendicular to the bilayer surface (the bilayer normal).

For the quantitative interpretation it is important to note that the two deuterons attached to the same segment give rise also to the same quadrupole splitting. This observation is non-trivial since in a completely rigid head group the individual deuterons would normally be inclined at different angles with respect to the rotation axis and would produce two separate quadrupole splittings. This is not borne out by the experiment, indicating a considerable configurational flexibility in the polar head group which averages out individual differences. The simplest mechanism in concord with the experimental observation would be 'a rapid equilibrium between two enantiomeric configurations, one with all torsion angles $\alpha_{i}$ positive, the other with all torsion angles negative. A transition from one configuration to the other would exchange the orientations of the corresponding deuterons. For a sufficiently rapid jump rate $\left(>10^{4} \mathrm{~Hz}\right)$ both deuterons would then experience the same average field gradient, leading to the same quadrupole splitting' (Seelig \& Gally, 1976). The rotation axis in this model is assumed to be the $\mathrm{C}(\mathrm{r})-\mathrm{C}(2)$ axis of the glycerol moiety. Two enantiomeric configurations have indeed been found in crystals of L- $\alpha$-glycerophosphorylcholine (Abrahamsson \& Pascher, I966), lending some experimental support to the above assumption.

The above results refer to fully hydrated dipalmitoyl-3-sn-phospha- 
tidylethanolamine. The hydration process itself may also be studied using deuterium magnetic resonance (Finer $\&$ Darke, 1974). If the lipid is hydrated with $\mathrm{D}_{2} \mathrm{O}$, at low water concentrations a spectrum containing a doublet of splitting up to $3 \mathrm{kHz}$ is observed due to the binding of the water molecules to the polar head. The spectrum has the shape characteristic of powder type spectra with the asymmetry parameter $\eta=0$. At increasing water contents the quadrupole splitting is replaced by a singlet indicative of free (bulk) water trapped between the lamellae but not strongly bound to the polar group. On the basis of these measurements it has been estimated that in bilayers of egg-yolk ethanolamine there exists only one hydration shell with about $\mathrm{I} 2$ water molecules bound per lipid molecule.

Considerable differences exist in the literature with respect to information on the orientation of the choline head group. Two extreme models have been proposed with the choline group being aligned either parallel or perpendicular to the bilayer surface (cf. Lee, 1975). However, more recent results seem to settle this dispute in favour of a parallel orientation of the choline group.

The most reliable X-ray diffraction study of a bilayer in the gel state is probably that of Blasie and co-workers (Lesslauer, Cain \& Blasie, I 972). Utilizing the surface deposition technique of Blodgett and Langmuir these authors prepared orientated multilayers of dipalmitoyl-3-sn-phosphatidylcholine and examined the $\mathrm{X}$-ray diffraction pattern in the range of relative humidities from 15 to $95 \%$. An electron density profile at about $6 \AA$ resolution was calculated which clearly showed that below the phase transition the phosphate and trimethylammonium groups lie in the same plane, so that the hydrated zwitterion must be oriented parallel to the bilayer surface.

In the liquid crystalline state the orientation of the head group cannot easily be determined using $\mathrm{X}$-ray diffraction. However, this problem has been overcome to a certain extent by measuring the distribution of heavy water using the neutron diffraction technique (Worcester \& Franks, 1976). It is concluded that the time-averaged position of the phosphorylcholine head group is approximately parallel to the surface of the bilayer for both dry and hydrated lecithin bilayers and also for bilayers of eggyolk lecithin containing cholesterol. The same studies also 'rule out the possibility of any appreciable amount of water uniformly distributed across the bilayer'.

Deuterium magnetic resonance studies have been performed with 


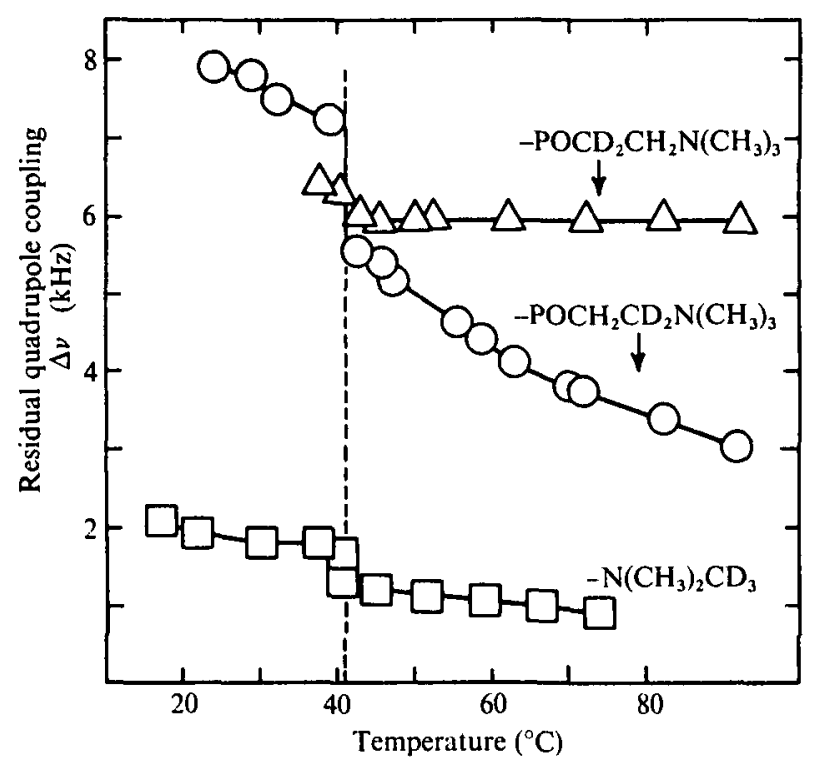

Fig. 22. Variation of residual quadrupole splittings of bilayers of deuterated dipalmitoyl-3-sn-phosphatidylcholine with temperature (5I.5 wt. \%water, 48.5 wt. \% lipid). (Gally et al. (1975), Biochemistry; reprinted with permission of the copyright owner.)

specifically deuterated dipalmitoyl-3-sn-phosphatidylcholine in order to elucidate details of the head group motion (Stockton et al. 1974; Gally et al. 1975; Seelig et al. 1977). In contrast to the results on phosphatidylethanolamine it is possible to observe well-resolved spectra of the choline deuterons at temperatures below the gel-to-liquid crystal phase transition. This leads to the conclusion that the molecular motions in the phosphatidylcholine head group are less restricted than are those in phosphatidylethanolamine. A similar result has been inferred from $\mathrm{nmr}$ studies of vesicles of bacterial phosphatidylethanolamine and phosphatidylcholine (Michaelson, Horwitz \& Klein, 1974). The temperature dependence of the deuterium quadrupole splittings of the three choline carbon atoms is shown in Fig. 22. A discontinuity at $4 \mathrm{I}^{\circ} \mathrm{C}$ in the otherwise smooth curves indicates the main transition, but no such change is observed at the pretransition at $34^{\circ} \mathrm{C}$. Similarly, if the phosphorus chemical shift anisotropy is measured as a function of temperature, only the main transition but not the pretransition is detected (Gally $e$ t al. 1975). These results suggest that the pretransition should be attributed not to the choline moiety but to the hydrocarbon chains (cf. previous section), 
as opposed to previous speculations which interpreted the pretransition as a conformational change in the polar group.

From studies with oriented multilayers of dipalmitoyl-3-sn-phosphatidylcholine it follows that the phosphorylcholine moiety like the phosphorylethanolamine group is rotating rapidly around an axis normal to the surface of the bilayer (Stockton et al. 1974; McLaughlin et al. 1975). Furthermore, each deuterated segment gives rise to only one quadrupole splitting which means that the individual deuterons attached to the same carbon atom experience the same movements. This is a strong indication for a rather flexible structure of the choline head group. Because the number of variable torsion angles $\left(\alpha_{1}-\alpha_{5}\right)$ exceeds the number of magnetic resonance parameters which are observed, the quantitative analysis of the extent of flexibility cannot be completely conclusive. In principle, the measured quadrupole splittings could be the averages of many different configurations of the phosphorylcholine group and it would then be impossible to assign one definite structure to the polar head group. However, it can be shown that the nmr data can also be explained quantitatively by the assumption of just two enantiomeric configurations (Seelig et al. 1977). The torsion angles evaluated from the $\mathrm{nmr}$ data are similar to those established for crystals of $\mathrm{L}-\alpha$-glycerophosphorylcholine which contain two enantiomeric molecules per unit cell (Abrahamsson \& Pascher, I966). If this model is correct, then the choline group as a whole is rotating around the $\mathrm{C}(\mathrm{I})-\mathrm{C}(2)$ axis of the glycerol backbone (nomenclature according to p. 406). This axis is oriented essentially perpendicular to the surface of the bilayer and has an order parameter of $c .0 \cdot 6-0.7$ as determined from the quadrupole splitting of a lipid deuterated at the glycerol-C(I) atom. The phosphodiester linkage is found to be in the gauche-gauche conformation which leads to an orientation of the choline group parallel to the surface of the bilayer. The $\mathrm{O}-\mathrm{C}-\mathrm{C}-\mathrm{N}$ system is also in the gauche state. This orientation of the choline head group is consistent with the $\mathrm{X}$-ray evidence for hydrated bilayers of lecithin and also with recent neutron diffraction studies using selectively deuterated lipids (Büldt et al. 1977).

Little information is available on the conformation of the glycerol backbone. Returning for a moment to Fig. ${ }_{7} \mathrm{C}$ it is seen that two splittings are observed if the deuterium label is attached to chain 2. This is unusual since two different quadrupole splittings are observed for the same $\mathrm{CD}_{2}$ group. One possibility to account for this effect would be to assume a rather rigid conformation of the glycerol backbone in which 
the two deuterium atoms are oriented at different angles with respect to the rotation axis. However, this interpretation seems not very probable since only one splitting shows a variation with temperature, whereas the other is temperature independent (Seelig \& Seelig, 1975, fig. 3). An alternative explanation would be the assumption of two long-lived conformations of the lipid molecule leading to two different orientations for the beginning of chain 2. The existence of two conformations of the glycerol constituent is further suggested by the fact that deuteration at carbon atom I (nomenclature of p. 406) of the glycerol backbone also produces two doublets. This would then mean that compared to the deuterium nmr time scale the conformational transitions are slow in the glycerol backbone and fast in the phosphorylcholine polar group. However, further experiments are required before a definite conclusion can be reached.

Due to sensitivity problems the use of deuterium magnetic resonance has so far been limited essentially to pure lipid systems. Recently the first applications of deuterium resonance to the study of a natural biological membrane have been described. Perdeuterated fatty acids (Oldfield, Chapman \& Derbyshire, 1972) and selectively deuterated palmitic acid (Stockton et al. 1975) were incorporated biosynthetically into the plasma membrane of Acholeplasma laidlawii and the deuterium quadrupole splitting could be measured as a function of temperature. Arvidson, Lindblom \& Drakenberg ( 1975 ) added deuterated choline to an otherwise choline-deficient diet of rats. By this method the rat liver cell membranes are labelled in vivo with deuterated choline which is essentially incorporated into lecithin and sphingomyelin. These results are encouraging since they show that deuterium magnetic resonance could become an important method also for the study of biological membranes.

This work was supported by the Swiss National Science Foundation grant no. $3 \cdot 390.74$ and grant 3.008 .76 .

\section{REFERENCES}

Abragam, A. (196r). The Principles of Nuclear Magnetism. London: Oxford University Press.

Abrahamsson, S. \& Pascher, I. (I966). Crystal and molecular structure of L- $\alpha$-glycerylphosphorylcholine. Acta crystallogr. 2r, 79-87.

Arvidson, G., Lindblom, G. \& Drakenderg, T. (1975). A novel approach to the study of mammalian cell membranes using deuterium nmr. FEBS Lett. 54, 249-52. 
BARNES, R. G. (1974). Deuteron quadrupole tensors in solids. In Advances in Nuclear Quadrupole Resonance, vol. I (ed. J. A. S. Smith), pp. 335-55. London: Heyden.

Barnes, R. G. \& Bloom, J. W. (1973). Deuteron quadrupole couplings in deuterated glycine. Molec. Phys. 25, 493-4.

Belle, J. \& Bothorel, P. (I974). Theoretical study of spin labeled aliphatic chains in bilayers. Biochem. biophys. Res. Commun. 58, 433-6.

Boden, N., Jackson, P., Levine, Y. K. \& WARD, A. J. I. (1976). Intramolecular disorder and its relation to mesophase structure in lipid/water mixtures. Biochim. biophys. Acta 419, 395-403.

Bothorel, P., Belle, J. \& Lemaire, B. (1974). Theoretical study of aliphatic chain structure in mono- and bilayers. Chem. Phys. Lipids 12, 96-1 16.

Brown, G. H., Doane, J. W. \& NefF, V. D. (I97I). A Review of the Structure and Physical Properties of Liquid Crystals. London: Butterworth.

Büldt, G., Zaccai, G., Seelig, J. \& Gally, H. (1977). (Manuscript in preparation.)

BURNETT, L. J. \& MülLER, B. H. (I97I). Deuteron quadrupole coupling constants in three solid deuterated paraffin hydrocarbons: $\mathrm{C}_{2} \mathrm{D}_{6}, \mathrm{C}_{4} \mathrm{D}_{10}$, $\mathrm{C}_{6} \mathrm{D}_{14} \cdot$. F. chem. Phys. 55, 5829-3 I.

Cadenhead, D. A. \& Müller-Landau, F. (1975). Monolayer studies of pure nitroxide fatty acid spin-label probes. Adv. Chem. Ser. 144, 294-307.

Caspary, W. J., Millett, F., Reichbach, M. \& Dailey, B. P. (ig69). NMR determination of deuterium quadrupole coupling constants in nematic solutions. 7 . chem. Phys. 5x, 623-7.

Chan, S. I., Seiter, C. H. A. \& Feigenson, G. W. (1972). Anisotropic and restricted molecular motion in lecithin bilayers. Biochem. biophys. Res. Commun. 46, $1488-92$.

Chan, S. I., Sheetz, M. P., Seiter, C. H. A., Feigenson, G. W., Hsu, M., LAU, A. \& YAU, A. (1973). Nuclear magnetic resonance studies of the structure of model membrane systems: The effect of surface curvature. Ann. N.Y. Acad. Sci., U.S.A. 222, 499-522.

Charvolin, J., Manneville, P. \& Deloche, B. (1973). Magnetic resonance of perdeuterated potassium laurate in oriented soap-water multilayers. Chem. Phys. Letters 23, 345-8.

ChiвA, T. (1962). Deuteron magnetic resonance study of several deuterated ammonium salts. $\mathcal{f}$. chem. Phys. 36, I 122-6.

Cohen, M. H. \& ReIF, F. (1957). Quadrupole effects in nuclear magnetic resonance studies of solids. Solid St. Phys. 5, 321-438.

Davis, J. H. \& JefFrey, K. R. (1977). The temperature dependence of chain disorder in potassium palmitate-water. A deuterium nmr study. Chem. Phys. Lipids (in the Press).

Derbyshire, W., Gorvin, T. \& Warner, D. (I969). A deuteron magnetic resonance study of a single crystal of deuterated malonic acid. Molec. Phys. 17, 401-7.

De TitTa, G. T. \& Craven, B. M. (I97I). Conformation of $O$-(L- $\alpha$-glyceryl- 
phosphoryl)-ethanolamine in the crystal structure of its monohydrate. Nature (Nerv Biol.) 233, 1 i 8-20.

De TitTA, G. T. \& CraveN, B. M. (I973). L- $\alpha$-Glycerylphosphorylethanolamine monohydrate. Acta crystallogr. B 29, $1354-7$.

Devaux, P. \& McConnell, H. M. (1972). Lateral diffusion in spin-labeled phosphatidylcholine multilayers. F. Am. chem. Soc. 94, 4475-81.

DieHL, P. (1974). A survey of recent advances in ${ }^{2} \mathrm{D}$ and ${ }^{3} \mathrm{~T}-\mathrm{NMR}$. In Nuclear Magnetic Resonance Spectra of Nuclei Other than Protons (ed. T. Axenrod and A. W. Graham), pp. 275-85. London: Wiley.

Diehl, P. \& Niederberger, W. (1974). Proton decoupling in deuteron magnetic resonance spectra of oriented molecules. $\mathcal{F}$. Magn. Reson. 15, $39 \mathrm{I}-2$.

Egozy, Y., Loewenstein, A. \& Silver, B. L. (1970). Deuterium relaxation in $\mathrm{C}_{6} \mathrm{D}_{6}$ dissolved in a liquid crystal. Molec. Phys. 19, 177-82.

Emsley, J. W., Lindon, J. C. \& TABONY, J. (I973a). NMR spectra of ethanol and partially deuterated ethanols as solutes in a nematic phase. Molec. Phys. 26, $1485-98$.

Emsley, J. W., Lindon, J. C. \& Tabony, J. (1973b). Measurement of deuterium quadrupole coupling constants of $\mathrm{CD}_{3}$ groups by ${ }^{1} \mathrm{H}-\left[{ }^{2} \mathrm{H}\right]$ NMR studies of nematic solutions. Molec. Phys. 26, I499-509.

FINER, E. G. (1973). Interpretation of deuteron magnetic resonance spectroscopic studies of the hydration of macromolecules. $\mathcal{F}$. Chem. Soc. Faraday Trans. II 69, I 590-600.

FINER, E. G. \& DARKe, A. (1974). Phospholipid hydration studied by deuteron magnetic resonance spectroscopy. Chem. Phys. Lipids 12, I-I6.

FloRy, P. J. (1969). Statistical Mechanics of Chain Molecules. New York: Interscience.

Fujiwara, F., Reeves, L. W., Tracey, A. S. \& Wilson, L. A. (I974). Studies of membrane processes. III. Deuterium magnetic resonance as a tool in studies of the lipophilic region of membrane systems. $\mathcal{F}$. Am. chem. Soc. 96, 5249-50.

Fung, B. M. (1974). Nuclear quadrupole coupling constants. In Critical Evaluation of Chemical and Physical Structural Information (D. R. Lide \& M. A. Paul), pp. 436-48. Washington, D.C. : National Academy of Sciences.

FUnG, B. M. \& WEI, I. Y. (1970). Proton and deuteron magnetic resonance of phenylsilane- $d_{3}$, phenylphosphine- $d_{2}$, and benzenethio-d in liquid crystal solutions. $\mathscr{F}$. Am. chem. Soc. 92, 1497-501.

Gaber, B. P. \& Peticolas, W. L. (1977). On the quantitative interpretation of biomembrane structure by Raman spectroscopy. Biochim. biophys. Acta 465, 260-74.

Gaffney, B. J. \& MCConnell, H. M. (1974). The paramagnetic resonance spectra of spin labels in phospholipid membranes. $\mathscr{F}$. Magn. Reson. 16, 1-28.

Gallot, B. \& Skoulious, A. (1966). Interactions électriques dans les phases mésomorphes des systemes amphiphile-eau: Rôle de la teneur en eau, de la longueur de la chaine paraffinique, de la nature du cation, et de la température. Kolloid Z. u. Z. Polymere 208, 37-43. 
Gally, H. U., Niederberger, W. \& Seelig, J. (I975). Conformation and motion of the choline head group in bilayers of dipalmitoyl-3-snphosphatidylcholine. Biochemistry, N.Y. 14, 3647-52.

Gally, H., Seelig, A. \& Seelig, J. (1976). Cholesterol induced rod-like motion of fatty acyl chains in lipid bilayers. A deuterium magnetic resonance study. Hoppe-Seyler's Z. physiol. Chem. 357, 1447-50.

Gray, G. W. \& Winsor, P. A. (1974). Liquid Crystals and Plastic Crystals. London: Wiley.

Henriksson, U., Oedberg, L. \& Eriksson, J. C. (1975). Quadrupole splittings in deuterium $\mathrm{nmr}$ spectra of the hexagonal phase in the system sodium octanoate- $\mathrm{d}_{15}$-water-carbontetrachloride. Mol. Cryst. Liquid Cryst. 30, 73-8.

Higgs, T. P. \& MACKaY, A. L. (I977). Determination of the complete order parameter tensor for a lipid methylene group from ${ }^{1} \mathrm{H}$ and ${ }^{2} \mathrm{H} \mathrm{nmr}$ spin labels. Chem. Phys. Lipids (in the Press).

Hitchcock, P. B., Mason, R., Thomas, K. M. \& Shipley, G. G. (1974). Structural chemistry of I,2-dilauroyl-DL-phosphatidylethanolamine: Molecular conformation and intermolecular packing of phospholipids. Proc. natn. Acad. Sci., U.S.A. 71, 3036-40.

Horwitz, A. F., Horsley, W. J. \& Klein, M. P. (I972). Magnetic resonance studies on membrane and model membrane systems: Proton magnetic relaxation rates in sonicated lecithin dispersions. Proc. natn. Acad. Sci., U.S.A. 69, 590-3.

Horwitz, A. F., Klein, M. P., Michaelson, D. M. \& Kohler, S. J. (I973). Magnetic resonance studies of membrane and model membrane systems. V. Comparisons of aqueous dispersions of pure and mixed phospholipids. Ann. N.Y. Acad. Sci., U.S.A. 222, 468-87.

Hoyland, J. R. (1968). Ab initio bond orbital calculations. I. Application to methane, ethane, propane and propylene. 7. Am. chem. Soc. 90, 2227-32.

Hubbell, W. L. \& McConnell, H. M. (I97I). Molecular motion in spin-labeled phospholipids and membranes. F. Am. chem. Soc. 93, 314-26.

HuNTRESS, W. T. (1970). The study of anisotropic rotation of molecules in liquids by quadrupolar relaxation. Adv. Magn. Reson. 4, 1-37.

Jacobs, R. E., Hudson, B. \& Andersen, H. C. (I975). A theory of the chain melting phase transition of aqueous phospholipid dispersions. Proc. natn. Acad. Sci., U.S.A. 72, 3993-7.

Jacobsen, J. P., Bildsoe, H. K. \& Schaumburg, K. (1976). Application of density matrix formalism in nmr spectroscopy. II. The one-spin-I case in anisotropic phase. 7 . Magn. Reson. 23, $153-64$.

Jacobsen, J.P. \& Schaumburg, K. (I976). Spin lattice relaxation time measurements of $\mathrm{D}_{2} \mathrm{O}$ in a lyotropic phase. $\mathcal{F}$. Magn. Reson. 24, 17380.

Janiak, M. J., Small, D. M. \& Shipley, G. G. (1976). Nature of the thermal pre-transition of synthetic phospholipids: dimyristoyl and dipalmitoyl lecithin. Biochemistry, N.Y. 15, 4575-80. 
Jost, P., Libertini, L. J., Hebert, V. C. \& Griffith, O. H. (1971). Lipid spin labels in lecithin multilayers. A study of motion along fatty acid chains. F. molec. Biol. 59, 77-98.

Kowalewski, J., Lindblom, T., Vestin, R. \& Drakenberg, T. (i976). Deuteron magnetic resonance of monodeuteroethene: Isotropic and anisotropic phase spectra. Molec. Phys. 31, 1669-76.

LeE, A. G. (r975). Functional properties of biological membranes: a physical chemical approach. Prog. Biophys. molec. Biol. 29, 3-56.

Lesslauer, W., Cain, J. E. \& Blasie, J. K. (1972). X-ray diffraction studies of lecithin bimolecular leaflets with incorporated fluorescent probes. Proc. natn. Acad., Sci. U.S.A. 69, 1499-503.

LipperT, J. L. \& Peticolas, W. L. (1971). Laser Raman investigation of the effect of cholesterol on conformational changes in dipalmitoyl lecithin multilayers. Proc. natn. Acad. Sci., U.S.A. 68, $1572-6$.

LoNG, R. C. \& GolDSTEIN, J. H. (1976). DMR investigation of alkyl chain order in an oriented quaternary potassium laurate mesophase. $\mathcal{f}$. Magn. Reson. 23, 519-22.

Luzzati, V. (1968). X-ray diffraction studies of lipid-water systems. In Biological membranes (ed. D. Chapman), pp. 7I-I23. New York: Academic Press.

MabReY, S. \& StURTEVANT, J. M. (1977). Incorporation of saturated fatty acids into phosphatidylcholine bilayers. Biochim. biophys. Acta 486, 444-50.

Mantsch, H. H., Saitô, H. \& Smith, I. C. P. (1977). Deuterium magnetic resonance. Applications in chemistry, physics and biology. In Progress in nmr Spectroscopy (ed. Emsley et al.). London: Pergamon Press. (In the Press.)

MARCELJA, S. (1974). Chain ordering in liquid crystals. II. Structure of bilayer membranes. Biochim. biophys. Acta 367, 165-76.

McCammon, J. A. \& Deutch, J. M. (1975). Semiempirical models for biomembrane phase transitions and phase separations. F. Am. chem. Soc. 97, 6675-8I.

MCCONNELL, H. M. (1976). Molecular motion in biological membranes. In Spin Labeling. Theory and Application (ed. L. J. Berliner), pp. 525-60. New York: Academic Press.

McConnell, H. M. \& McFarland, B. G. (1972). The flexibility gradient in biological membranes. Ann. N.Y. Acad. Sci., U.S.A. 195, 207-17.

McFarland, B. G. \& McConnell, H. M. (1971). Bent fatty acid chains in lecithin bilayers. Proc. natn. Acad. Sci., U.S.A. 68, $1274-8$.

Mclaughlin, A. C., Cullis, P. R., Hemminga, M. A., Houldt, D. I., Radda, G. K., Ritchie, G. A., Seeley, P. J. \& Richards, R. E. (1975). Application of ${ }^{31} \mathrm{P} \mathrm{nmr}$ to model and biological membrane systems. FEBS Lett. 57, 213-17.

Mely, B. \& Charvolin, J. (1977). DMR comparison of lyotropic mesophases with ordered and disordered paraffinic chains. Chem. Phys. Lipids 19, $43-55$. 
Mely, B., Charvolin, J. \& Keller, P. (1975). Disorder of lipid chains as a function of their lateral packing in lyotropic liquid crystals. Chem. Phys. Lipids 15, 161-73.

MENDELSOHN, R. (1972). Laser Raman spectroscopic study of egg lecithin and egg lecithin-cholesterol mixtures. Biochim. biophys. Acta 290, I5-2 I.

Metcalfe, J. C., Birdsall, N. J. M., Feeney, J., Lee, A. G., Levine, Y. K. \& Partington, P. (I971). ${ }^{13} \mathrm{C}$ NMR spectra of lecithin vesicles and erythrocyte membranes. Nature, Lond. 233, I99-201.

Metcalfe, J. C., Birdsall, N. J. M. \& LeE, A. G. (1973). NMR studies of lipids in bilayers and membranes. Ann. N.Y. Acad. Sci., U.S.A. 222, $460-7$.

Michaelson, D. M., Horwitz, A. F. \& Klein, M. P. (i974). Head group modulation of membrane fluidity in sonicated phospholipid dispersions. Biochemistry, N.Y. 13, 2605-15.

NAGLE, J. F. (1973). Theory of biomembrane phase transitions. fo. chem. Phys. 58, 252-64.

NiederberGer, W. \& SeELiG, J. (1974). Deuterium-magnetische Resonanzspektroskopie an spezifisch deuterierten flüssigen Kristallen. Ber. Bunsenges. physik. Chem. 78, 947-9.

Niederberger, W. \& Seelig, J. (1976). Phosphorus-3 I chemical shift anisotropy in unsonicated phospholipid bilayers. F. Am. chem. Soc. 98, 3704-6.

Oldfield, E., Chapman, D. \& Derbyshire, W. (1971). Deuteron resonance: a novel approach to the study of hydrocarbon chain mobility in membrane systems. FEBS Lett. 16, 102-4.

Oldfield, E., Chapman, D. \& Derbyshire, W. (1972). Lipid mobility in Acholeplasma membranes using deuteron magnetic resonance. Chem. Phys. Lipids 9, 69-8I.

Pechнold, W. (I968). Molekülbewegung in Polymeren. Kolloid-Z. u. Z. Polymere 228, $\mathrm{I}-38$.

Pines, A., Ruben, D. J., Vega, S. \& Mehring, M. (1976). New approach to high-resolution $\mathrm{nmr}$ in solids: deuterium spin-decoupling by multiple quantum transitions. Phys. Rev. Lett. 36, 1 10-13.

Rand, P. R., Chapman, D. \& Larsson, K. (1975). Tilted hydrocarbon chains of dipalmitoyllecithin become perpendicular to the bilayer before melting. Biophys. F. 15, II 17-24.

ReEves, L. W. \& Tracey, A. S. (I975). Studies of membrane processes. VII. Hydrocarbon chain motions and the effect of changing counter-ions. F. Am. chem. Soc. 97, 5729-35.

REID, R. V. \& VAIDA, M. L. (I972). Quadrupole moment of the deuteron. Phys. Rev. Lett. 29, 494-6.

Rose, M.E.(1957). Elementary Theory of Angular Momentum. New York: Wiley. RoseVEaR, F. B. (1968). Liquid crystals: The mesomorphic phases of surfactant compositions. $\mathcal{F}$. Soc. Cosmetic Chem. 19, $5^{81-94 .}$

Rowell, J. C., Phillips, W. D., Melby, L. R. \& Panar, M. (1965). NMR studies of some liquid crystal systems. F. chem. Phys. 43, 3442-54.

Saitô, H., Schreier-Muccillo, S. \& Smith, I. C. P. (1973). High resolution 
deuterium magnetic resonance - an approach to the study of molecular organization in biological membranes and model systems. FEBS Lett. $33,28 I-5$.

SAUPE, A. (1964). Kernresonanzen in kristallinen Flüssigkeiten und kristallinflüssigen Lösungen. $Z$. Naturf. 19a, 16 I-71.

SchindleR, H. \& SeElig, J. (I973). EPR spectra of spin labels in lipid bilayers. F. chem. Phys. 59, I84I-50.

Schindler, H. \& SeElig, J. (I974). EPR spectra of spin labels in lipid bilayers. II. Rotation of steroid spin probes. F. chem. Phys. 6r, 2946-9.

SchiNDLER, H. \& SeElig, J. (I975). Deuterium order parameters in relation to thermodynamic properties of a phospholipid bilayer. A statistical mechanical interpretation. Biochemistry, N.Y. 14, 2283-7.

ScotT, H. L. (1974). A model for phase transitions in lipid bilayers and biological membranes. F. theor. Biol. 46, 24I-53.

Seelig, J. (1970). Spin label studies of oriented liquid crystals (A model system for bilayer membranes). F. Am. chem. Soc. 92, 388 I-7.

SeELIG, J. (1976). Anisotropic motion in liquid crystalline structures. In Spin Labeling: Theory and Application (ed. L. J. Berliner), pp. 373-409. New York: Academic Press.

Seelig, J. \& Gally, H. (1976). Investigation of phosphatidylethanolamine bilayers by deuterium and phosphorus-3 $\mathrm{I}$ nuclear magnetic resonance. Biochemistry, N.Y. 15, 5199-204.

Seelig, J., Gally, H. \& Wohlgemuth, R. (1977). Orientation and flexibility of the choline head group in lecithin bilayers. Biochim. biophys. Acta 467 , 109-119.

Seelig, J., Limacher, H. \& Bader, P. (1972). Molecular architecture of liquid crystalline bilayers. f. Am. chem. Soc. 94, 6364-71.

Seelig, J. \& Niederberger, W. (1974a). Deuterium labeled lipids as structural probes in liquid crystalline bilayers. F. Am. chem. Soc. 96, 2069-72.

Seelig, J. \& Niederberger, W. (1974 $b$ ). Two pictures of a lipid bilayer. A comparison between deuterium label and spin label experiments. Biochemistry, N.Y. 13, $1585-8$.

Seelig, J. \& Seelig, A. (1974a). Deuterium magnetic resonance studies of phospholipid bilayers. Biochem. biophys. Res. Commun. 57, 406-I I.

Seelig, A. \& Seelig, J. (1974b). The dynamic structure of fatty acyl chains in a phospholipid bilayer measured by deuterium magnetic resonance. Biochemistry, N.Y. 13, 4839-45.

Seelig, A. \& Seelig, J. (1975). Bilayers of dipalmitoyl-3-sn-phosphatidylcholine. Conformational differences between the fatty acyl chains. Biochim. biophys. Acta 406, I-5.

Seelig, A. \& Seflig, J. (1977). Effect of a single cis-double bond on the structure of a phospholipid bilayer. Biochemistry, N.Y. 16, 45-50.

Slichter, C. P. (1963). Principles of Magnetic Resonance, pp. 160-76. New York: Harper and Row.

SodA, G. \& CHIBA, T. (1969). Deuteron magnetic resonance study of cupric sulfate pentahydrate. $\mathcal{F}$. chem. Phys. 50, 439-55. 
Stockton, G. W. \& Sмıтн, I. C. P. (1976). A deuterium magnetic resonance study of the condensing effect of cholesterol on egg phosphatidylcholine bilayer membranes. I. Perdeuterated fatty acid probes. Chem. Phys. Lipids 17, $25 \mathrm{I}-63$.

Stockton, G. W., Polnaszek, C. F., Leitch, L. C., Tulloch, A. P. \& Smith, I. C. P. (1974). A study of mobility and order in model membranes using ${ }^{2} \mathrm{H} \mathrm{nmr}$ relaxation rates and quadrupole splittings of specifically deuterated lipids. Biochem. biophys. Res. Commun. 6o, 844-50.

Stockton, G. W., Polnaszek, C. F., Tulloch, A. P., Hasan, F. \& Smith, I. C. P. (1976). Molecular motion and order in single-bilayer vesicles and multilamellar dispersions of egg lecithin and lecithin cholesterol mixtures. A deuterium nuclear magnetic resonance study of specificallylabelled lipids. Biochemistry, N.Y. 15, 954-66.

Stockton, G. W., Johnson, K. G., Butler, K. W., Polnaszek, C. F., Cyr, R. \& SMIth, I. C. P. (I975). Molecular order in Acholeplasma laidlawii membranes as determined by deuterium magnetic resonance of biosynthetically incorporated specifically-labelled lipids. Biochim. biophys. Acta 401, 535-9.

Sundaralingam, M. (1972). Molecular structures and conformations of the phospholipids and sphingomyelins. Ann. N.Y. Acad. Sci., U.S.A. I95, $324-55$.

Tardieu, A., Luzzatr, V. \& Reman, F. C. (1973). Structure and polymorphism of the hydrocarbon chains of lipids: A study of lecithin-water phases. F. molec. Biol. 75, 7 I I-33.

Townes, C. H. \& Schawlow, A. L. (1955). Microwave Spectroscopy, pp. 130-40. New York: McGraw-Hill.

TräUble, H. \& Sackmann, E. (1972). Studies of the crystalline-liquid crystalline phase transition of lipid model membranes. III. Structure of a steroid-lecithin system below and above the lipid phase transition. f. Am. chem. Soc. 94, 4499-510.

TraceY, A. S. \& DieHL, P. (1976). A deuterium magnetic resonance study of a lamellar lyotropic liquid crystalline phase. Can. F. Chem. 54, 2283-7.

VAughan, D. J. \& Keough, K. M. (1974). Changes in phase transition of phosphatidylethanolamine - and phosphatidylcholine - water dispersions induced by small modifications in the head group and backbone region. FEBS Lett. 47, $158-6 \mathrm{r}$.

VEGA, S. \& Pines, A. (1977). Operator formalism for double quantum $\mathrm{nmr}$. f. chem. Phys. (in the Press).

Wennerström, H., Persson, N. O. \& Lindman, B. (i974). Double quantum transitions in deuteron $\mathrm{nmr}$ spectra of lyotropic liquid crystals. $\mathcal{F}$. Magn. Reson. 13, 348-53.

WENNERSTRÖM, H. \& LiNDBlom, G. (1977). Biological and model membranes studied by nuclear magnetic resonance of spin one half nuclei. $Q$. Rev. Biophys. ro, 67-96.

WORCESTER, D. L. \& FRANKS, N. P. (1976). Neutron diffraction analysis of hydrated egg lecithin and cholesterol bilayers. F. molec. Biol. 100, 359-78. 